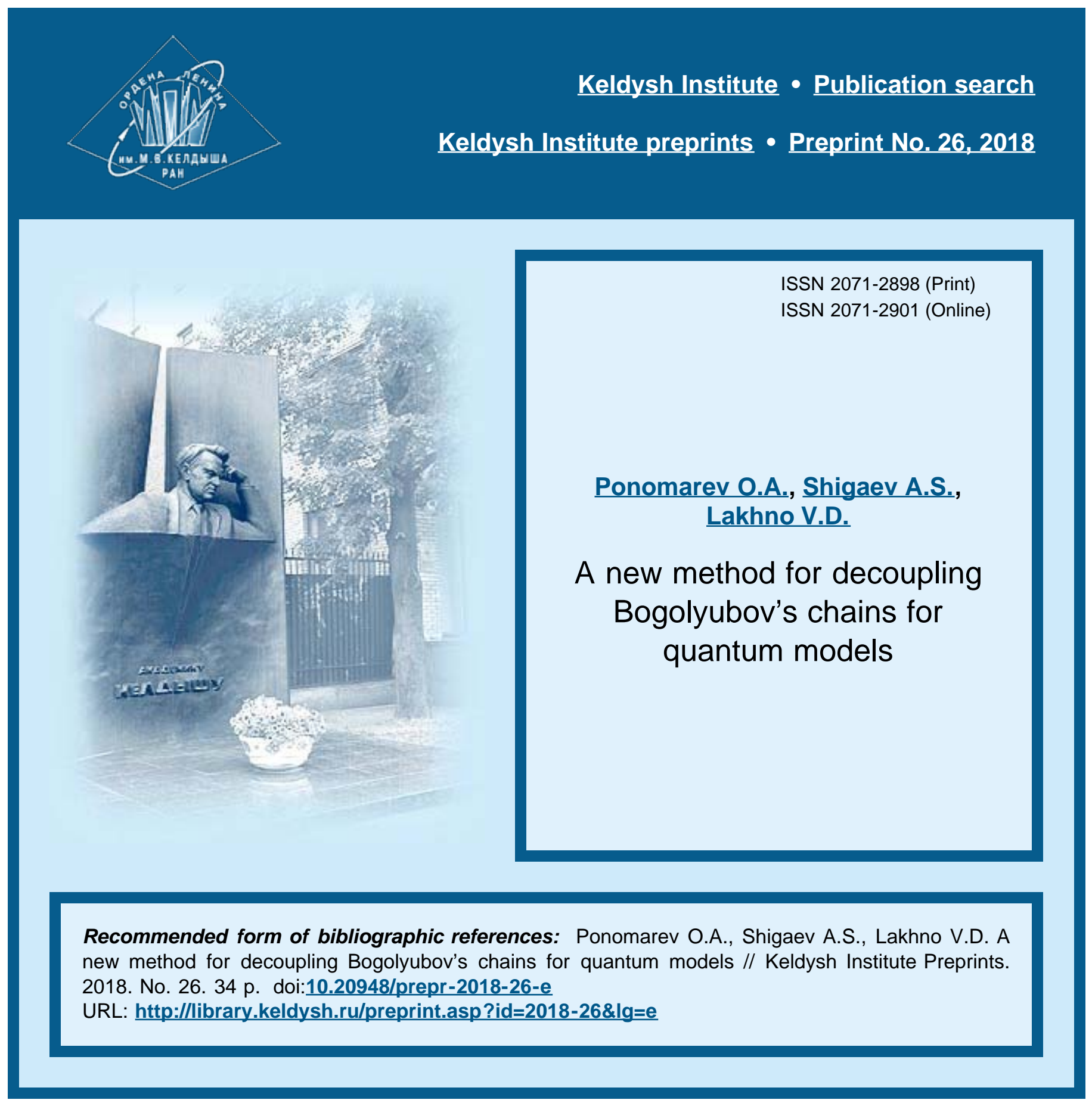




$$
\begin{gathered}
\text { О р д е н а Л н и н а } \\
\text { ИНСТИТУТ ПРИКЛАДНОЙ МАТЕМАТИКИ } \\
\text { имени М.В.Келдыша } \\
\text { Р о с и й с к й а к а д м и и н а к }
\end{gathered}
$$

O.A. Ponomarev, A.S. Shigaev, V.D. Lakhno

\section{A new method for decoupling Bogolyubov's chains for quantum models}


Пономарёв О.А., Шигаев А.С., Лахно В.Д.

Новый метод обрыва цепочек Боголюбова для квантовых моделей

В данной работе подробно описан метод исследования эффектов динамической связи в процессах переноса энергии и квантовых частиц с учётом усреднённого внешнего поля всех частиц системы. В рамках метода введено ограничение парными корреляциями для взаимодействующих частиц, позволяющее эффективный обрыв цепочек Боголюбова. Показано, что данный метод позволяет эффективный учёт температуры и хорошо подходит для большинства квантовомеханических систем, где маловероятны тройные корреляции квантовых частиц. Возможности подхода наглядно продемонстрированы на примерах исследования моделей Мацубары, Фрёлиха и Юкавы.

Ключевые слова: расцепление цепочек Боголюбова, корреляционные функции, функции Грина, приближение парных корреляций

\section{Oleg Aleksandrovich Ponomarev, Alexey Sergeevich Shigaev, Victor Dmitrievich Lakhno}

\section{A new method for decoupling Bogolyubov's chains for quantum models}

The paper describes in detail a method for investigating the effects of dynamical coupling in the energy- and charge-transfer processes with regard to an averaged external field of all the particles of the system. The particles are assumed to take part only in pair correlations which makes possible effective truncation of Bogolubov's chains. It is shown that the method enables effective consideration of temperature and is well suited for most of quantum-mechanical systems where triple correlations of quantum particles are hardly probable. To demonstrate the potentialities of the approach the models by Matsubara, Froehlich and Yukawa are investigated.

Key words: Bogolyubov chains' decoupling, correlation functions, Green's functions, approximation of pair correlations

Работа выполнена при поддержке Российского фонда фундаментальных исследований, проект 16-07-00305-а. 


\section{Introduction}

Analytical investigation of quantum models of various physical and chemical systems is one of the most complicated problems of the present-day physics. The vital role in its solution belongs to general methods of modern statistical physics, the most fruitful of which are the methods of correlation functions (CF) and Green's functions. Various variants of the last were developed by Bogolyubov's school. The main results are presented in papers [ $1-7]$. Some important findings in this field can also be found in paper by Peletminsky [8].

It is well known that the methods of CF and Green's functions lead to a system of coupled linear equations. For most of nontrivial systems their number is infinite. Therefore, to solve the problem one should reduce the number of equations so that to make it finite. This is achieved in two ways. The first way is to choose the "critical" order of CF followed by omitting all higher-order CF. The second one is approximate expression of higher-order $\mathrm{CF}$ in terms of lower-order $\mathrm{CF}$. In both the cases an infinite system of linear equations becomes a finite one which can easily be solved.

Such approximations are suitable only for systems with weak dynamical coupling. Besides, CF obtained by such methods describe properly the asymptotic behavior of molecular systems only for long-range time. Another drawback of these two ways of truncating a chain of equations is that they cannot be used in studies of systems with intermediate and strong dynamical coupling. The main contribution into $\mathrm{CF}$ in these cases is made by a region of relatively short-range time.

Besides, the change-over to a finite system of linear equations produces a serious risk that some effective correlations between the modes can be lost in the course of calculations. This may lead to erroneous results. In this context of importance is development of an effective method for truncating Bogolubov's chains in which the risk of error is minimum.

The paper describes in detail a new method of decoupling Bogolyubov's chains equations for quantum models. The method is suitable for investigating the effects of dynamical coupling in the energy- and charge-transfer processes with regard to an averaged external field of all the particles of the system in the short-range timescale. Moreover, the method enables one to assess the measure of inaccuracy of the solutions obtained. For neighboring particles, we restrict ourselves only by pair correlations. It is shown that the method is highly effective for the study of quantum models, in particular in the cases when the temperature contribution should be taken into account.

The method presented is new. It is well suited for investigation of systems with strong interaction since the perturbation theory is based not on the interaction force but on the number of interacting particles. Therefore in small-size systems the method has proved to be rather efficient $[9,10]$. To demonstrate its reliability the models by Matsubara, Froehlich and Yukawa are investigated.

The paper is arranged as follows. In Section 2 we describe the method and determine the models for which its application gives exact results. In Section 3 the method is used to investigate the Matsubara model. The Matsubara model simulates a 
one dimensional system of states without the conductivity band which contains a few charge carriers [11]. This method was earlier developed by us for the case of charge transfer in DNA [10]. The perturbation theory in this case is constructed relying on the width of the conductivity band (exchange integral).

In Section 4 we study the interaction between two nucleons in the model by Yukawa. It has turned out that our method readily leads to an exact result which reproduces those obtained by $\mathrm{N}$. Mugibayashi [12] with the help of a much more laborious method (using Feynman diagrams, see [13]).

In Section 5 consideration is given to a two-level model of non-adiabatic chemical reactions. In this case we can restrict ourselves by the second order of the perturbation theory with respect to exchange integral. In this approximation the method proposed also yields exact results. This enables one to develop the perturbation theory with respect to exchange integral.

The method developed is well suited for systems with any dynamical coupling provided that triple correlations are hardly probable. In our method insignificant correlations are automatically excluded in the course of formation of a system of relevant differential equations. The physical meaning of this approximation is to consider accurately pair correlations and exclude triple ones. In the course of analytical investigation it turned out that the method has an intrinsic criterion of accuracy. This enables one to determine the limits of its applicability for various molecular systems.

The authors believe that the wide use of their method can provide obtaining new results and finding new models solvable by this method.

\section{General formalism of the method}

Since the approach under discussion is universal it makes sense to express its formalism for an arbitrary Hamiltonian of the form

$$
H=H\left(a, a^{+}, b, b^{+}\right),
$$

where $a$ is a set of electron operators $a_{n}, b$ is a set of boson operators $b_{q} ; a^{+}$and $b^{+}$ stand for Hermite-conjugate operators $a$ and $b$. Let us consider the correlation function

$$
G(t)=<A(t) B(0)>, \quad t>0,
$$

where $A(t)$ is a product of an arbitrary number of operators $a_{n}, a_{n}^{+}$and $b_{q}, b_{q}^{+}$, depending on time $t ; B(0)$ is the same or a different product of operators at zero moment of time. The angle brackets $<\ldots>$ symbolize averaging with the density matrix

$$
\rho=\rho^{\prime} \exp \{-\beta(H-F)\} / \operatorname{Sp}\left(\exp \{-\beta(H-F)\} \rho^{\prime}\right)
$$




$$
\rho^{\prime}=T \exp \left\{\sum \int_{0}^{\infty} u_{q_{\alpha}}^{\alpha}(t) z_{q_{\alpha}}^{\alpha}(t) d t\right\} .
$$

where $F$ is the Helmholtz free energy, $u_{q_{\alpha}}^{\alpha}$ are external auxiliary classical fields, $T$ is the operator of chronological ordering, $\beta$ is the reciprocal temperature. For compactness of the notation, we use the designation

$$
z_{q_{1}}^{1}=a_{n}^{+}, z_{q_{2}}^{2}=a_{n}, z_{q_{3}}^{3}=b_{q}^{+}, z_{q_{4}}^{4}=b_{q} .
$$

Having taken the variational derivative of expression (2) with respect to field $u_{q_{\alpha}}^{\alpha}\left(t_{1}\right)$ we have

$$
\frac{\delta<T A(t) B(0)>}{\delta u_{q_{\alpha}}^{\alpha}\left(t_{1}\right)}=<T z_{q_{\alpha}}^{\alpha}\left(t_{1}\right) A(t) B(0)>-<z_{q_{\alpha}}^{\alpha}\left(t_{1}\right)><T A(t) B(0)>
$$

or

$$
<T z_{q_{\alpha}}^{\alpha}\left(t_{1}\right) A(t) B(0)>=\left[<z_{q_{\alpha}}^{\alpha}\left(t_{1}\right)>+\frac{\delta}{\delta u_{q_{\alpha}}^{\alpha}\left(t_{1}\right)}\right]<T A(t) B(0)>.
$$

Expression (3) makes possible reducing the order of CF by introducing variational derivatives with respect to external auxiliary classical fields. The procedure can be continued. Let us replace an arbitrary number of operators by a relevant number of variational derivatives. Having acted on both sides of the last expression by the operator

$$
<z_{q_{\beta}}^{\beta}\left(t_{2}\right)>+\frac{\delta}{\delta u_{q_{\beta}}^{\beta}\left(t_{2}\right)},
$$

we get

$$
\begin{gathered}
<T z_{q_{\alpha}}^{\alpha}\left(t_{1}\right) z_{q_{\beta}}^{\beta}\left(t_{2}\right) A(t) B(0)>= \\
=\left[<z_{q_{\alpha}}^{\alpha}\left(t_{1}\right)>+\frac{\delta}{\delta u_{q_{\alpha}}^{\alpha}\left(t_{1}\right)}\right]\left[<z_{q_{\beta}}^{\beta}\left(t_{2}\right)>+\frac{\delta}{\delta u_{q_{\beta}}^{\beta}\left(t_{2}\right)}\right]<T A(t) B(0)>.
\end{gathered}
$$

This equality is exact. In the general case we have

$$
<T \prod_{i=1}^{N} z_{q i}^{i}\left(t_{i}\right) A(t) B(0)>=\prod_{i=1}^{N}\left[<z_{q_{i}}^{i}\left(t_{i}\right)>+\frac{\delta}{\delta u_{q_{i}}^{i}\left(t_{i}\right)}\right]<T A(t) B(0)>,
$$

Now let us determine the new functional $M_{q_{\alpha}}^{\alpha}\left(t_{1} ; t\right)$ by the relation 


$$
\begin{gathered}
<T z_{q_{\alpha}}^{\alpha}\left(t_{1}\right) A(t) B(0)>=M_{q_{\alpha}}^{\alpha}\left(t_{1} ; t\right)<T A(t) B(0)>= \\
=\left[<z_{q_{\alpha}}^{\alpha}\left(t_{1}\right)>+\frac{\delta}{\delta u_{q_{\alpha}}^{\alpha}\left(t_{1}\right)}\right]<T A(t) B(0)>= \\
=<T A(t) B(0)>\left[M_{q_{\alpha}}^{\alpha}\left(t_{1} ; t\right)+\frac{\delta}{\delta u_{q_{\alpha}}^{\alpha}\left(t_{1}\right)}\right] \cdot 1 .
\end{gathered}
$$

Convenience of this formal notation will be seen in the subsequent discussion.

The last part of this equality is an identity. Let us consider it in greater detail:

$$
\left[<z_{q_{\alpha}}^{\alpha}\left(t_{1}\right)>+\frac{\delta}{\delta u_{q_{\alpha}}^{\alpha}\left(t_{1}\right)}\right]<T A(t) B(0)>=<T A(t) B(0)>\left[M_{q_{\alpha}}^{\alpha}\left(t_{1} ; t\right)+\frac{\delta}{\delta u_{q_{\alpha}}^{\alpha}\left(t_{1}\right)}\right] \cdot 1 .
$$

Having acted on both sides of this expression by operator (4) we get

$$
\begin{aligned}
& {\left[<z_{q_{\alpha}}^{\alpha}\left(t_{1}\right)>+\frac{\delta}{\delta u_{q_{\alpha}}^{\alpha}\left(t_{1}\right)}\right]\left[<z_{q_{\beta}}^{\beta}\left(t_{2}\right)>+\frac{\delta}{\delta u_{q_{\beta}}^{\beta}\left(t_{2}\right)}\right]<T A(t) B(0)>=} \\
= & {\left[<z_{q_{\alpha}}^{\alpha}\left(t_{1}\right)>+\frac{\delta}{\delta u_{q_{\alpha}}^{\alpha}\left(t_{1}\right)}\right]<T A(t) B(0)>\left[M_{q_{\beta}}^{\beta}\left(t_{2} ; t\right)+\frac{\delta}{\delta u_{q_{\beta}}^{\beta}\left(t_{2}\right)}\right] \cdot 1=} \\
& <T A(t) B(0)>\left[M_{q_{\alpha}}^{\alpha}\left(t_{1} ; t\right)>+\frac{\delta}{\delta u_{q_{\alpha}}^{\alpha}\left(t_{1}\right)}\right]\left[M_{q_{\beta}}^{\beta}\left(t_{2} ; t\right)+\frac{\delta}{\delta u_{q_{\beta}}^{\beta}\left(t_{2}\right)}\right] \cdot 1 .
\end{aligned}
$$

In the general case

$$
\prod_{i=1}^{N}\left[<z_{q_{i}}^{i}\left(t_{i}\right)>+\frac{\delta}{\delta u_{q_{i}}^{i}\left(t_{i}\right)}\right]<T A(t) B(0)>=<T A(t) B(0)>\prod_{i=1}^{N}\left[M_{q_{i}}^{i}\left(t_{i} ; t\right)+\frac{\delta}{\delta u_{q_{i}}^{i}\left(t_{i}\right)}\right] \cdot 1 .
$$

Taking (5) into account, we get the exact relation

$$
<T \prod_{i=1}^{N} z_{q_{\alpha_{i}}}^{\alpha_{i}}\left(t_{i}\right) A(t) B(0)>=<T A(t) B(0)>\Pi\left[M_{q_{\alpha_{i}}}^{\alpha_{i}}\left(t_{i} ; t\right)+\frac{\delta}{\delta u_{q_{\alpha_{i}}}^{\alpha_{i}}\left(t_{i}\right)}\right] \cdot 1 .
$$

This is the most important relation which is central to all subsequent calculations. It is a new representation of $\mathrm{CF}$ of arbitrary rank. Approximate representation of higher-order $\mathrm{CF}$ in terms of lower-order ones is chosen just on the basis of this relation. 
Without being related to any particular Hamiltonian, this approximation of $\mathrm{CF}$ is monotypic for any model. The main point of the approximation is that in the righthand side of equation (6) there remain only the terms with functionals

$$
M_{q_{\alpha_{i}}}^{\alpha_{i}}\left(t_{i} ; t\right) \quad \text { and } \quad D_{q_{\alpha_{i}} q_{\alpha_{j}}}^{\alpha_{i} \alpha_{j}}\left(t_{i}, t_{j} ; t\right)=\frac{\delta M_{q_{\alpha_{j}}}^{\alpha_{j}}\left(t_{j} ; t\right)}{\delta u_{q_{\alpha_{i}}}^{\alpha_{i}}\left(t_{i}\right)},
$$

determined implicitly. Derivatives $M_{q_{\alpha_{i}}}^{\alpha_{i}}\left(t_{i} ; t\right)$ with respect to $u_{q}^{\alpha}(t)$ of the second and higher orders are omitted, since we carry out investigations approximately only with regard to pair correlations.

If we write down expression (6) in the form

$$
<T z^{N} A(t) B(0)>=<A(t) B(0)>\left[M+\frac{\delta}{\delta u}\right]^{N} \cdot 1 .
$$

then the approximate decoupling will be:

$$
<T z^{N} A(t) B(0)>\approx<A(t) B(0)>\left[M^{N}+(N-1) \frac{\delta M}{\delta u} M^{N-2}\right] \cdot 1,
$$

where the symbol «₹» signifies the error of this approximation.

Then the problem is reduced to determining $M$ and $\delta M / \delta u=D$, which depend on the form of the system; they are different for different Hamiltonians and determined from dynamical equations. To determine $M$ and $D$ exactly let us use motion equations for operators. Suppose $A(t)=z_{q_{\alpha}}^{\alpha}(t)$. For this operator, motion equation has the form:

$$
i \frac{d}{d t} z_{q_{\alpha}}^{\alpha}(t)=\left[z_{q_{\alpha}}^{\alpha}(t), H\right]
$$

Suppose that the right-hand side of this expression can be presented in the form:

$$
\left[z_{q_{\alpha}}^{\alpha}(t), H\right]=\sum_{q_{\alpha}^{1}} F_{q_{\alpha} q_{\alpha}^{1}}^{\alpha}\left(a, a^{+}, b, b^{+}\right) z_{q_{\alpha}^{1}}^{\alpha}(t) .
$$

where $F_{q_{\alpha} q_{\alpha}^{\prime}}^{\alpha}\left(a, a^{+}, b, b^{+}\right)$are the well-known operator functions. Then the expression for $\mathrm{CF}$ is written as: 


$$
\begin{aligned}
& i \frac{d}{d t}<z_{q_{\alpha}}^{\alpha}(t) B(0)>=\sum_{q_{\alpha}^{1}}<F_{q_{\alpha} q_{\alpha}^{1}}^{\alpha}\left(\ldots, z_{q_{\gamma}}^{\gamma}(t), \ldots\right) z_{q_{\alpha}^{1}}^{\alpha}(t) B(0)>=, \\
& \quad=\sum_{q_{\alpha}^{1}}<z_{q_{\alpha}^{1}}^{\alpha}(t) B(0)>F_{q_{\alpha} q_{\alpha}^{1}}^{\alpha}\left(\ldots, M_{q_{\gamma}}^{\gamma}(t ; t)+\frac{\delta}{\delta u_{q_{\gamma}}^{\gamma}(t)}, \ldots\right) \cdot 1,
\end{aligned}
$$

Exact equality (8) is obtained with regard for expression (6) without any approximations.

Now let us describe the way of closing the system of equations of Bogolubov's chain. For this purpose we should write down expressions for functional $M_{q_{\alpha}}^{\alpha}(t ; t)$. In this case it is convenient to use the equation

$$
\begin{gathered}
i \frac{d}{d t}\left[M_{q_{\beta}}^{\beta}(t ; t)<z_{q_{\alpha}}^{\alpha}(t) B(0)>\right]=i \frac{d}{d t}<z_{q_{\beta}}^{\beta}(t) z_{q_{\alpha}}^{\alpha}(t) B(0)>=, \\
=\sum_{q_{\beta}^{1}}<z_{q_{\alpha}}^{\alpha}(t) B(0)>F_{q_{\beta} q_{\beta}^{1}}^{\beta}\left(\ldots, M_{q_{\gamma}}^{\gamma}(t ; t)+\frac{\delta}{\delta u_{q_{\gamma}}^{\gamma}(t)}, \ldots\right)\left(M_{q_{\beta}^{1}}^{\beta}(t ; t)+\frac{\delta}{\delta u_{q_{\beta}}^{\beta}(t)}\right) \cdot 1+, \\
+\sum_{q_{\alpha}^{1}}<z_{q_{\alpha}^{1}}^{\alpha}(t) B(0)>F_{q_{\alpha} q_{\alpha}^{1}}^{\alpha}\left(\ldots, M_{q_{\gamma}}^{\gamma}(t ; t)+\frac{\delta}{\delta u_{q_{\gamma}}^{\gamma}(t)}, \ldots\right)\left(M_{q_{\beta}}^{\beta}(t ; t)+\frac{\delta}{\delta u_{q_{\beta}}^{\beta}(t)}\right) \cdot 1 .
\end{gathered}
$$

When (9) is transformed to approximation (7) its right-hand side will contain only functionals $M, D$ and $G$. Now the problem is reduced to determining the functional $D$. To do this we write down the equation: 


$$
\begin{gathered}
i \frac{d}{d t}<z_{q_{\gamma}}^{\gamma}(t) z_{q_{\beta}}^{\beta}(t) z_{q_{\alpha}}^{\alpha}(t) B(0)>= \\
=i \frac{d}{d t}\left\{<z_{q_{\alpha}}^{\alpha}(t) B(0)>\left(M_{q_{\gamma}}^{\gamma}(t ; t) M_{q_{\beta}}^{\beta}(t ; t)+D_{q_{\gamma} q_{\beta}}^{\gamma \beta}(t, t ; t)\right)\right\}= \\
=\sum_{q_{\gamma}^{1}}<z_{q_{\alpha}}^{\alpha}(t) B(0)>F_{q_{\gamma} q_{\gamma}^{1}}^{\gamma}\left(\ldots, M_{q_{\delta}}^{\delta}(t ; t)+\frac{\delta}{\delta u_{q_{\delta}}^{\delta}(t)}, \ldots\right)\left(M_{q_{\gamma}^{1}}^{\gamma}(t ; t)+\right. \\
\left.+\frac{\delta}{\delta u_{q_{\gamma}^{1}}^{\gamma}(t)}\right)\left(M_{q_{\beta}}^{\beta}(t ; t)+\frac{\delta}{\delta u_{q_{\beta}}^{\beta}(t)}\right) \cdot 1+ \\
+\sum_{q_{\beta}^{1}}<z_{q_{\alpha}^{1}}^{\alpha}(t) B(0)>F_{q_{\beta} q_{\beta}^{1}}^{\beta}\left(\ldots, M_{q_{\delta}}^{\delta}(t ; t)+\frac{\delta}{\delta u_{q_{\delta}}^{\delta}(t)}, \ldots\right)\left(M_{q_{\gamma}}^{\gamma}(t ; t)+\right. \\
\left.+\frac{\delta}{\delta u_{q_{\gamma}}^{\gamma}(t)}\right)\left(M_{q_{\beta}}^{\beta}(t ; t)+\frac{\delta}{\delta u_{q_{\beta}^{\prime}}^{\beta}(t)}\right) \cdot 1+ \\
+\sum_{q_{\alpha \gamma}^{1}}<z_{q_{\alpha}}^{\alpha}(t) B(0)>F_{q_{\alpha} q_{\alpha}^{1}}^{\alpha}\left(\ldots, M_{q_{\delta}}^{\delta}(t ; t)+\frac{\delta}{\delta u_{q_{\delta}}^{\delta}(t)}, \ldots\right)\left(M_{q_{\gamma}}^{\gamma}(t ; t)+\right. \\
\left.+\frac{\delta}{\delta u_{q_{\gamma}}^{\gamma}(t)}\right)\left(M_{q_{\beta}}^{\beta}(t ; t)+\frac{\delta}{\delta u_{q_{\beta}}^{\beta}(t)}\right) \cdot 1 .
\end{gathered}
$$

Bearing in mind approximation (7), we find that in this approximation there also remain only functionals $M, D$ and $G$ with various indices. Nevertheless the action of the operators $\delta / \delta u$ on these functionals enable us to pass on to the limit $u \rightarrow 0$ and solve the system (8)-(10) not for the functionals but for functions.

The initial conditions for relevant equations can be found from the relations of the form

$$
\begin{gathered}
G(0) \pm G(-i \beta)=[A(0), B(0)]_{ \pm} \quad u=0 \\
i \frac{d}{d t} G(t) \pm i \frac{d}{d t} G(t-i \beta)=\left[[A(0), H]_{-}, B(0)\right]_{ \pm} \quad u=0 \quad t=0 .
\end{gathered}
$$

etc.

For Hamiltonian (1), if we successively reduce the number of operators in CF we can get a number in its right-hand side. In this case we have to solve systems like (8)-(10) for relevant $\mathrm{CF}$ of lower order. For more complicated systems we should determine the average values of relevant commutators.

There exists another way of getting equations for functionals $M$ and $D$, which sometimes appears to be more convenient. It implies determining $M$ via $<z_{q_{\alpha}}^{\alpha}(t) B(0)>$ in the form 


$$
\begin{gathered}
M_{q_{\gamma}}^{\gamma}\left(t_{1} ; t\right)=<z_{q_{\gamma}}^{\gamma}\left(t_{1}\right)>+\frac{\delta}{\delta u_{q_{\gamma}}^{\gamma}} \ln <z_{q_{\alpha}}^{\alpha}(t) B(0)>, \\
D_{q_{\gamma} q_{\beta}}^{\gamma \beta}\left(t_{1}, t_{2} ; t\right)=\frac{\delta M_{q_{\beta}}^{\beta}\left(t_{2} ; t\right)}{\delta u_{q_{\gamma}}^{\gamma}\left(t_{1}\right)}=<\left(z_{q_{\gamma}}^{\gamma}\left(t_{1}\right)-<z_{q_{\gamma}}^{\gamma}\left(t_{1}\right)>\right)\left(z_{q_{\beta}}^{\beta}\left(t_{2}\right)-<z_{q_{\beta}}^{\beta}\left(t_{2}\right)>\right)>+ \\
+\frac{\delta}{\delta u_{q_{\gamma}}^{\gamma}\left(t_{1}\right)} \frac{\delta}{\delta u_{q_{\beta}}^{\beta}\left(t_{2}\right)} \ln <z_{q_{\alpha}}^{\alpha}(t) B(0)>.
\end{gathered}
$$

In this approach, initial conditions can also be obtained from commutation relations. In the subsequent text the technique presented here will be used for simple mathematical models of lattice structures. It will be shown that for some models our method provides results to a good approximation and in some cases - exact solutions. On the other hand, the use of particular models enables us to elucidate more thoroughly the peculiarities of our method and reveal the limits of its applicability. In what follows we will also demonstrate the physical meaning of our approximation more precisely.

\section{The use of the method for generalized Matsubara model}

The first model to which our method will be applied is generalized Matsubara model [11] which is a particular case of a commonly known one-dimensional Holstein approach. Hamiltonian of Matsubara model has the form:

$$
H=\sum E_{n} a_{n}^{+} a_{n}+\sum \omega_{q} b_{q}^{+} b_{q}+\sum A_{q n} r_{q} a_{n}^{+} a_{n}, \quad r_{q}=b_{q}+b_{q}^{+},
$$

The distinctive feature of Matsubara model is the possibility of its analytical solution. Therefore it is an especially suitable instrument for testing the method under discussion.

Besides, the model is intuitively clear and has easily understandable physical meaning. It corresponds to the problem of electron interaction with a phonon field in zero approximation with respect to the width of the conductivity band. Matsubara Hamiltonian is a limit case of Fröhlich Hamiltonian for zero overlapping integral: $I \rightarrow 0$. Therefore this model suits well to test our method.

The spectral densities of absorption and irradiation of the probability of nonradiative nonadiabatic processes as well as the probabilities of electron transfer in a one-dimensional molecular lattice are conveniently expressed in terms of $\mathrm{CF}$. In calculations of these CF it is sufficient to take account of the second order with respect to the exchange interaction in view of negligibly small probability of triple correlations. Therefore, in finding CF for calculating the constants of the chemical reactions rates of the first order in a one-dimensional lattice, a real Fröhlich-type Hamiltonian can also be replaced by Matsubara Hamiltonian. 
Actually, collisions of more than two quantum particles in a one-dimensional system are practically impossible if spaced apart sites of a one-dimensional chain do not contact with one another in 3D space. Moreover, if such a contact is possible, these collisions are extremely improbable too. Therefore this approximation can be considered to be exact.

To determine the electronic spectrum of the system described by Hamiltonian (13) we should calculate CF in the form:

$$
G_{m n}(t)=S p\left\{\exp \{\beta(F-H)\} a_{m}^{+}(t) a_{n}(0)\right\}=<a_{m}^{+}(t) a_{n}(0)>_{0} .
$$

Closed equations in functional derivatives are exact and therefore are suitable for various approximations. To construct these equations let us consider a generalized $\mathrm{CF}$ :

$$
G_{m n}(t)=S p\left\{\rho a_{m}^{+}(t) a_{n}(0)\right\}=<a_{m}^{+}(t) a_{n}(0)>
$$

where

$$
\rho=\exp \{\beta \cdot(F-H)\} \rho^{1} /<\rho^{1}>_{0}, \quad \rho^{1}=T \exp \left\{\int_{0}^{\infty} \sum_{q} u_{q}(t) r_{q}(t) d t\right\} .
$$

For this $\mathrm{CF}$, we have retained the same denotation as for any correlation functions described above. For $u_{q} \rightarrow 0$ expression (15) takes the same form as expression (14). Introduction of classical fields $u_{q}$ into the definition of $\mathrm{CF}$ enables one, with the use of classical differentiation, to close formally the chain of equations for any correlation function (see, for example [5]). Indeed, a motion equation for functions like $G_{n m}(t)$ will have the form

$$
\frac{d}{d t} G_{n m}=i E_{n} G_{n m}+i \sum_{q} A_{q n}<r_{q}(t) a_{n}^{+}(t) a_{m}(0)>,
$$

Having taken the variational derivative of $G_{n m}(t)$ with respect to $u_{q}$, we get

$$
\frac{\delta G_{n m}(t)}{\delta u_{q}\left(t_{1}\right)}=<r_{q}\left(t_{1}\right) a_{n}^{+}(t) a_{m}(0)>-<r_{q}\left(t_{1}\right)>G_{n m}(t)
$$

or

$$
<r_{q}\left(t_{1}\right) a_{n}^{+}(t) a_{m}(0)>=\left[<r_{q}\left(t_{1}\right)>+\frac{\delta}{\delta u_{q}\left(t_{1}\right)}\right] G_{n m}(t) .
$$

Substituting (17) into (16), we get a "closed" equation 


$$
\frac{d}{d t} G_{n m}=i E_{n} G_{n m}+i \sum_{q} A_{q n}\left[\left\langle r_{q}(t)\right\rangle+\frac{\delta}{\delta u_{q}(t)}\right] G_{n m}(t) .
$$

Let us rewrite it in the integral form. To do this we introduce a new function $M_{q}\left(t_{1} ; t, n m\right)$ :

$$
\left[<r_{q}\left(t_{1}\right)>+\frac{\delta}{\delta u_{q}\left(t_{1}\right)}\right] G_{n m}(t)=G_{n m}\left[M_{q}\left(t_{1} ; t, n m\right)+\frac{\delta}{\delta u_{q}\left(t_{1}\right)}\right] \cdot 1,
$$

Having substituted (19) for $t_{1}=t$ into (18) and performed integration we get an integral form of equation (19):

$$
G_{n m}(t)=G_{n m}(0) \exp \left\{i E_{n} t+i \int_{0}^{t} \sum A_{q n} M_{q}\left(t_{1} ; t_{1}, n m\right) d t_{1}\right\} .
$$

This equation together with expression (19) for $M$ forms a closed system of functional equations. Earlier it was shown [14], that

$$
<T \prod_{i=1}^{N} r_{q_{i}}\left(t_{i}\right) a_{n}^{+}(t) a_{m}(0)>=G_{n m}(t) \prod_{i=1}^{N}\left\{M_{q_{i}}\left(t_{i} ; t, n m\right)+\frac{\delta}{\delta u_{q_{i}}\left(t_{i}\right)}\right\} \cdot 1 .
$$

This equality enables one to express CF of higher order in terms of $M$ and variational derivatives of $M$. We will believe that

$$
\delta^{k} M / \delta u_{q_{1}} \ldots \delta u_{q_{k}}=0, \quad k>1 .
$$

In approximation (21) we get

$$
\begin{gathered}
<r_{q}\left(t_{1}\right) a_{n}^{+}(t) a_{m}(0)>=G_{n m}(t) M_{q}\left(t_{1} ; t, n m\right), \\
<T r_{q}\left(t_{1}\right) r_{q_{1}}\left(t_{2}\right) a_{n}^{+}(t) a_{m}(0)>= \\
=G_{n m}(t)\left[M_{q}\left(t_{1} ; t, n m\right) M_{q_{1}}\left(t_{2} ; t, n m\right)+D_{q q_{1}}\left(t_{1}, t_{2} ; t, n m\right)\right], \\
<T_{q_{1}}\left(t_{1}\right) r_{q_{2}}\left(t_{2}\right) r_{q_{3}}\left(t_{3}\right) a_{n}^{+}(t) a_{m}(0)>= \\
=G_{n m}(t)\left[M_{q_{1}}\left(t_{1} ; t, n m\right) M_{q_{2}}\left(t_{2} ; t, n m\right) M_{q_{3}}\left(t_{3} ; t, n m\right)+\right. \\
+M_{q_{1}}\left(t_{1} ; t, n m\right) D_{q_{2} q_{3}}\left(t_{2}, t_{3} ; t, n m\right)+ \\
\left.+M_{q_{2}}\left(t_{2} ; t, n m\right) D_{q_{1} q_{3}}\left(t_{1}, t_{3} ; t, n m\right)+M_{q_{3}}\left(t_{3} ; t, n m\right) D_{q_{1} q_{2}}\left(t_{1}, t_{2} ; t, n m\right)+\ldots\right],
\end{gathered}
$$


where

$$
D_{q q_{1}}\left(t_{1}, t_{2} ; t, n m\right)=\delta M_{q_{1}}\left(t_{2} ; t, n m\right) / \delta u_{q}\left(t_{1}\right) .
$$

is causal Green's function, see $[3,4]$.

Decoupling (21) reduces an infinite system of linear equations to a finite system of nonlinear ones. The functional $M_{q}\left(t_{1} ; t, n m\right)$ is determined by functions $D_{q q_{1}}\left(t_{1}, t_{2} ; t, n m\right)$. At the same time no other functions are introduced in the theory. Therefore approximation (21) means that we are limited by the case when the oscillatory system is completely described by pair CF. This property is peculiar to only Gaussian systems: in all other cases it is an approximation whose validity should be checked.

From definition of functional $M_{q}\left(t_{1} ; t, n m\right)$ by formula (19) we get:

$$
M_{q}\left(t_{1} ; t, n m\right)=<r_{q}\left(t_{1}\right)>+\frac{\delta}{\delta u_{q}\left(t_{1}\right)} \ln G_{n m}(t)
$$

Expressing $G_{n m}(t)$ via $M_{q}\left(t_{1} ; t, n m\right)$, we have

$$
M_{q}\left(t_{1} ; t, n m\right)=<r_{q}\left(t_{1}\right)>+i \int_{0}^{t} d t_{2} \sum_{q_{1}} A_{q_{1} n} \frac{\delta M_{q_{1}}\left(t_{2} ; t_{2}, n m\right)}{\delta u_{q_{1}}\left(t_{1}\right)}+\frac{\delta \ln G_{n m}(0)}{\delta u_{q_{1}}\left(t_{1}\right)} .
$$

Let us consider the functional derivative (26). For our Hamiltonian $G_{n m}(0)=G_{n n}(0) \delta_{n m}$. Let us consider the expression

$$
\begin{gathered}
{\left[\begin{array}{c}
q \\
q
\end{array}\left(t_{1}\right)>+\frac{\delta}{\delta u_{q}\left(t_{1}\right)}\right] G_{n n}(0)=G_{n n}(0) M_{q}\left(t_{1} ; 0, n n\right)=<r_{q}\left(t_{1}\right) a_{n}^{+}(0) a_{n}(0)>=} \\
=<r_{q}\left(t_{1}\right) a_{n}^{+}\left(t_{1}\right) a_{n}\left(t_{1}\right)>=<r_{q}(0) a_{n}^{+}(0) a_{n}(0)>=M_{q}(0 ; 0, n n) G_{n n}(0)
\end{gathered}
$$

In deriving this expression we used the property of generalized Matsubara model $a_{n}^{+}(0) a_{n}(0)=a_{n}^{+}\left(t_{1}\right) a_{n}\left(t_{1}\right)$. Hence, $M_{q}\left(t_{1} ; 0, n n\right)=M_{q}(0 ; 0, n n)$.

To determine functions $M_{q}(0 ; 0, n n)$ let us consider the expression $\left(b_{q}^{-} \equiv b_{q}\right)$

$$
\begin{gathered}
i \frac{d}{d t}<b_{q}^{ \pm}(t) a_{n}^{+}(t) a_{n}(t)>=0= \pm \omega_{q}<b_{q}^{ \pm}(t) a_{n}^{+}(t) a_{n}(t)> \pm \\
\pm \sum A_{q p}<a_{p}^{+}(t) a_{p}(t) a_{n}^{+}(t) a_{n}(t)>.
\end{gathered}
$$

Since the approximation of a small density of quantum particles is assumed to be fulfilled, then

$$
<a_{p}^{+}(t) a_{p}(t) a_{n}^{+}(t) a_{n}(t)>=<a_{n}^{+} a_{n}>\delta_{p n},
$$


and one can readily see that

$$
M_{q}(0 ; 0, n n)=-\frac{2 A_{q n}}{\omega_{q}}=<r_{q}>\text { and } \frac{\delta G_{n n}(0)}{\delta u_{q}(t)}=0 .
$$

Now expression (26) takes the form

$$
M_{q}\left(t_{1} ; t, n m\right)=<r_{q}\left(t_{1}\right)>+i \int_{0}^{t} d t_{2} \sum_{q_{1}} A_{q_{1} n} \frac{\delta M_{q_{1}}\left(t_{2} ; t_{2}, n m\right)}{\delta u_{q_{1}}\left(t_{1}\right)} .
$$

Accordingly, for a pair CF we have:

$$
D_{q_{1} q_{2}}\left(t_{1}, t_{2} ; t, n m\right)=\frac{\delta M_{q_{1}}\left(t_{1} ; t, n m\right)}{\delta u_{q_{2}}\left(t_{2}\right)}=\frac{\delta M_{q_{2}}\left(t_{2} ; t, n m\right)}{\delta u_{q_{1}}\left(t_{1}\right)}=\frac{\delta<r_{q_{1}}\left(t_{1}\right)>}{\delta u_{q_{2}}\left(t_{2}\right)} .
$$

The quantities $M_{q}\left(t_{1} ; t, n m\right)$ and $D_{q q_{1}}\left(t_{1}, t_{2} ; t, n m\right)$ are the new and main objects in the theory, in terms of which all the characteristics of the studied system are expressed. Therefore it makes sense to elucidate their physical meaning in detail.

It follows from the definition of the function $M_{q}\left(t_{1} ; t, \mathrm{~nm}\right)$ that it describes a certain effective field which acts on the aforementioned particles. This function has some peculiarities. It depends on the type of the system, the form of CF it belongs to and the energy of interaction between the particles. Besides, $M_{q}\left(t_{1} ; t, n m\right)$ depends on the temperature, on the time $t$ which characterizes the state of the electron subsystem and also on the oscillatory time $t_{1}$.

Functions $D_{q q_{1}}\left(t_{1}, t_{2} ; t, \mathrm{~nm}\right)$ are generalizations of causal Green's functions of phonons. They depend on the electron time $\mathrm{t}$ and two oscillatory times $t_{1}$ and $t_{2}$ correspondingly. These functions also depend on the form of $\mathrm{CF}$, parameters of the Hamiltonian and the temperature.

The physical meaning of the suggested approximation is that the indirect interaction between bosons caused by their interaction via electrons is considered to be small. The explicit form of the function $D_{q q_{1}}\left(t_{1}, t_{2} ; t, n m\right)$ in the case of linear interaction after passing on to the limit $u_{q} \rightarrow 0$ is:

$$
\begin{gathered}
D_{q_{1} q_{2}}\left(t_{1}, t_{2} ; t, n m\right)=\frac{\delta<r_{q_{1}}\left(t_{1}\right)>}{\delta u_{q_{2}}\left(t_{2}\right)}=\left(<\operatorname{Tr}_{q_{1}}\left(t_{1}\right) r_{q_{2}}\left(t_{2}\right)>-\right. \\
\left.-<r_{q_{1}}\left(t_{1}\right)><r_{q_{2}}\left(t_{2}\right)>\right)=D_{q_{1} q_{2}}^{0}\left(t_{1}-t_{2}\right)
\end{gathered}
$$

where $D_{q_{1} q_{2}}^{0}\left(t_{1}-t_{2}\right)$ is the causal Green's function of the phonon subsystem.

To determine the explicit form of $D_{q_{1} q_{2}}^{0}\left(t_{1}-t_{2}\right)$ we will write down a differential equation for it with the use of the equations for operators 


$$
i \frac{d}{d t} r_{q}=\omega_{q}\left(b_{q}-b_{-q}^{+}\right) \quad, \quad i \frac{d}{d t}\left(b_{q}-b_{-q}^{+}\right)=\omega_{q} r_{q}+2 \sum_{n} A_{q n} a_{n}^{+} a_{n} .
$$

and the expression

$$
a_{n}^{+}(t) a_{n}(t)=\text { const } .
$$

For this purpose we twice differentiate $D_{q_{1} q_{2}}^{0}\left(t_{1}-t_{2}\right)$ with respect to time and get:

$$
\left(i \frac{d}{d t}\right)^{2} D_{q_{1} q_{2}}^{0}(t)=\omega_{q_{1}}^{2} D_{q_{1} q_{2}}^{0}(t)-2 i \omega_{q_{1}} \delta(t),
$$

The initial conditions for this expression are calculated straightforwardly and equal to

$$
D_{q_{1} q_{2}}^{0}(0)=\left(2 N_{q_{1}}+1\right) \delta\left(q_{1}+q_{2}\right), \quad i \frac{d}{d t} D_{q_{1} q_{2}}^{0}(t \rightarrow 0)=\delta\left(q_{1}+q_{2}\right) .
$$

The solution of equation (29) is a function

$$
D_{q_{1} q}^{0}(t)=\left[N_{q} \exp \left\{i \omega_{q} t\right\}+\left(N_{q}+1\right) \exp \left\{-i \omega_{q} t\right\}\right] \delta\left(q+q_{1}\right), \quad t \geq 0 .
$$

Substituting it into (27), we have for $u_{q}=0$ :

$$
\begin{gathered}
M_{q}\left(t_{1} ; t, n m\right)=-2 \frac{A_{-q n}}{\omega_{q}}+ \\
+\frac{A_{q n}}{\omega_{q}}\left[\exp \left\{i \omega_{q} t_{1}\right\} N_{q}\left(1-\exp \left\{-i \omega_{q} t\right\}\right)+\exp \left\{-i \omega_{q} t_{1}\right\}\left(N_{q}+1\right)\left(\exp \left\{i \omega_{q} t_{1}\right\}-1\right)\right] .
\end{gathered}
$$

Putting in this expression $t_{1}=t$ we have:

$$
M_{q}(t ; t, n m)=-\frac{A_{-q n}}{\omega_{q}}\left[-1+N_{q} \exp \left\{i \omega_{q} t\right\}-\left(N_{q}+1\right) \exp \left\{-i \omega_{q} t\right\}\right] .
$$

Substituting (29) into (20) and performing integration we obtain the expression

$$
\begin{gathered}
G_{n m}(t)=G_{n m}(0) \delta_{n m} \exp \left\{i E_{n} t-i \sum \frac{A_{q n}^{2}}{\omega_{q}} t+\right. \\
\left.+\sum \frac{A_{q n}^{2}}{\omega_{q}^{2}}\left[N_{q}\left(e^{i \omega_{q} t}-1\right)+\left(N_{q}+1\right)\left(e^{-i \omega_{q} t}-1\right)\right]\right\} .
\end{gathered}
$$

It is specific that, expression (31) coincides with the exact result obtained by summation of all Feynman diagrams. 
Even if there were not an exact solution of Matsubara model one could prove that conditions (7) and (21) are automatically fulfilled for it [15]. To do this no special assumption is needed. In order to make sure that this is the case let us write down equations for higher variational derivatives of $M_{q}\left(t_{1} ; t, n m\right)$ with respect to $u$ and calculate them with the use of Wick's theorem [16]. On the assumption that the system contains $N$ phonon particles let us write down an equation for $\delta^{N} M / \delta u^{N}=0$. Then it follows from functional equations that $\delta^{N-1} M / \delta u^{N-1}=0$; all the previous terms up to $\delta^{2} M / \delta u^{2}$ are also equal to zero [9]. Hence the suggested decoupling is exact for the model under consideration.

In conclusion let us take a brief look at Fröhlich model for small-radius polarons [17]. This model as well as some more complicated ones is considered in detail in [18]. Fröhlich model is widely used in solid state physics and can present both the limit cases of the system: stochastic and dynamic ones. It enables one to consider theoretically the motion of a hole (or electron) over a DNA fragment with due regard for the relaxation process $[19,20]$ and calculate the yield of luminescence upon recombination of an anion-radical with a cation-radical. The recombination time reflects the hole path and depends on the fragment structure [21, 22]. The decoupling considered in the previous section is approximate for Fröhlich model described by Hamiltonian in impulse representation:

$$
H=\sum E_{k} a_{k}^{+} a_{k}+\sum \omega_{q} b_{q}^{+} b_{q}+A_{q} a_{k+q}^{+} a_{k} r_{q} .
$$

In what follows we will restrict ourselves to the case of small density of electrons which is justified in considering semiconductors. Let us consider the electron spectrum of this system. For this purpose we should calculate causal Green's function of the form

$$
G_{k k_{1}}(t)=<T a_{k}^{+}(t) a_{k_{1}}(0)>.
$$

Upon bringing the function to the diagonal form its poles will determine the spectrum of excitations.

Recall that in our case the symbol $<\ldots>$ means averaging with regard to $\rho^{1}$ which includes external fields $u_{q}(t)$. Therefore functions $G_{k k_{1}}$ are nondiagonal with respect to $k$ and $k_{1}$; for $u_{q}(t)=0$ they become diagonal automatically.

Formally a closed functional equation, by analogy with (18), has the form

$$
\frac{d}{d t} G_{k k_{1}}(t)=i E_{k} G_{k k_{1}}+i \sum A_{q}\left(<r_{q}>+\frac{\delta}{\delta u_{q}(t)}\right) G_{k+q k_{1}}(t) \quad t \geq 0 .
$$

Let us rewrite it in the integral form: 


$$
\begin{gathered}
G_{k k_{1}}(t)=\exp \left\{i E_{k} t\right\}\left(G_{k k_{1}}(0) \delta_{k k_{1}}+\right. \\
\left.+i \int_{0}^{t} d t_{2} \exp \left\{-i E_{k} t_{2}\right\} \sum A_{q_{2}}\left[<r_{q_{2}}>+\frac{\delta}{\delta u_{q_{2}}\left(t_{2}\right)}\right] G_{k+q_{2} k_{1}}\left(t_{2}\right)\right) .
\end{gathered}
$$

Having acted on both the sides of this equation by the operator

$$
\left[<r_{q_{1}}\left(t_{1}\right)>+\frac{\delta}{\delta u_{q_{1}}\left(t_{1}\right)}\right],
$$

we have:

$$
\begin{gathered}
{\left[<r_{q_{1}}\left(t_{1}\right)>+\frac{\delta}{\delta u_{q_{1}}\left(t_{1}\right)}\right] G_{k k_{1}}(t)=G_{k k_{1}}(t) M_{q_{1}}\left(t_{1} ; t, k, k_{1}\right)=} \\
=e^{i E_{k} t}\left\{\left[<r_{q_{1}}\left(t_{1}\right)>+\frac{\delta}{\delta u_{q_{1}}\left(t_{1}\right)}\right] G_{k k_{1}}(0) \delta_{k k_{1}}+\right. \\
\left.+i \int_{0}^{t} d t_{2} e^{-i E_{k} t_{2}} \sum A_{q_{2}}\left[<r_{q_{1}}\left(t_{1}\right)>+\frac{\delta}{\delta u_{q_{1}}\left(t_{1}\right)}\right]\left[<r_{q_{2}}\left(t_{2}\right)>+\frac{\delta}{\delta u_{q_{2}}\left(t_{2}\right)}\right] G_{k+q_{2} k_{1}}\left(t_{2}\right)\right\}= \\
=e^{i E_{k} t}\left\{\left[<r_{q_{1}}\left(t_{1}\right)>+\frac{\delta}{\delta u_{q_{1}}\left(t_{1}\right)}\right] G_{k k_{1}}(0) \delta_{k k_{1}}+\right. \\
+i \int_{0}^{t} d t_{2} e^{-i E_{k} t_{2}} \sum A_{q_{2}} G_{k+q_{2} k_{1}}\left(t_{2}\right)\left[M_{q_{1}}\left(t_{1} ; t, k+q_{2}, k_{1}\right)+\right. \\
\left.\left.+\frac{\delta}{\delta u_{q_{1}}\left(t_{1}\right)}\right] M_{q_{2}}\left(t_{2} ; t, k+q_{2}, k_{1}\right)\right\}= \\
=e^{i E_{k} t}\left\{\left[<r_{q_{1}}\left(t_{1}\right)>+\frac{\delta}{\delta u_{q_{1}}\left(t_{1}\right)}\right] G_{k k_{1}}(0) \delta_{k k_{1}}+\right. \\
+i \int_{0}^{t} d t_{2} e^{-i E_{k} t_{2}} \sum A_{q_{2}} G_{k+q_{2} k_{1}}\left(t_{2}\right)\left[M_{q_{1}}\left(t_{1} ; t, k+q_{2}, k_{1}\right) M_{q_{2}}\left(t_{2} ; t, k+q_{2}, k_{1}\right)+\right. \\
\left.\left.+D_{q_{1} q_{2}}\left(t_{1}, t_{2} ; t, k+q_{2}, k_{1}\right)\right]\right\},
\end{gathered}
$$

where

$$
D_{q_{1} q_{2}}\left(t_{1}, t_{2} ; t, \ldots\right)=\delta M_{q_{1}}\left(t_{1} ; t, \ldots\right) / \delta u_{q_{2}}\left(t_{2}\right)=\delta M_{q_{2}}\left(t_{2} ; t, \ldots\right) / \delta u_{q_{1}}\left(t_{1}\right),
$$

Assessing the terms involved in (34) we get from (32) 


$$
M_{q} \approx\left(A_{q} / \omega_{q}\right)=\gamma_{q}, \quad D_{q q_{1}} \approx\left(2 N_{q_{1}}+1\right) \delta\left(q+q_{1}\right)
$$

and also

$$
<r_{q}>\approx\left(A_{q} / \omega_{q}\right)=\gamma_{q}, \quad \delta G / \delta u_{q} \approx \gamma_{q} G, \quad \delta^{2} G / \delta u^{2} \approx \gamma^{2} G .
$$

Assuming $\gamma_{q}<<1$, we obtain from (35)

$$
D_{q_{1} q_{2}}\left(t_{1}, t_{2} ; t, \ldots\right)=\delta<r_{q_{1}}\left(t_{1}\right)>/ \delta u_{q_{2}}\left(t_{2}\right)=D_{q_{1} q}^{0}\left(t_{1}-t_{2}\right) \delta\left(q_{1}+q_{2}\right), \quad u \rightarrow 0 .
$$

where

$$
D_{q_{1} q}^{0}(t)=\left[N_{q} \exp \left\{i \omega_{q} t\right\}+\left(N_{q}+1\right) \exp \left\{-i \omega_{q} t\right\}\right] \delta\left(q+q_{1}\right), \quad t \geq 0 .
$$

is the causal function of free bosons.

Functions

$$
M_{q}\left(t_{1} ; t, k+q, k_{1}\right) G_{k+q k_{1}}(t) .
$$

are obtained from (34) for $\left\langle r_{q}\right\rangle=0$ :

$$
\begin{gathered}
M_{q}\left(t_{1} ; t, k+q, k_{1}\right) G_{k+q k_{1}}(t)= \\
=i \exp \left\{i E_{k+q} t\right\} \int_{0}^{t_{1}} d t_{2} \exp \left\{-i E_{k+q} t_{2}\right\} D_{q}^{0}\left(t_{1}-t_{2}\right) A_{-q} G_{k k_{1}}\left(t_{2}\right) .
\end{gathered}
$$

Substituting this expression into (33) we get the integral equation

$$
\begin{gathered}
G_{k k_{1}}(t)=\exp \left\{i E_{k} t\right\}\left\{G_{k k_{1}}(0) \delta_{k k_{1}}+\right. \\
\left.+i \int_{0}^{t} d t_{1} \sum \exp \left\{-i\left(E_{k}-E_{k+q}\right) t_{1}\right\} A_{q}^{2} \int_{0}^{t_{1}} d t_{2} \exp \left\{-i E_{k+q} t_{2}\right\} D_{q}^{0}\left(t_{1}-t_{2}\right) G_{k k_{1}}\left(t_{2}\right)\right\},
\end{gathered}
$$

To solve this integral equation let us pass on to an equivalent system of differential equations. Having differentiated (36) with respect to time we get:

$$
\begin{gathered}
i \frac{d}{d t} G_{k k_{1}}=-E_{k} G_{k k_{1}}-\sum A_{q}^{2}\left(N_{q}+1\right) P_{k k_{1} q}^{(1)}-\sum A_{q}^{2} N_{q} P_{k k_{1} q}, \\
G_{k k_{1}}(0)=G_{k}^{0} \delta_{k k_{1}},
\end{gathered}
$$

where

$$
P_{k k_{1} q}=i \exp \left\{i\left(E_{k+q}-\omega_{q}\right) t\right\} \int_{0}^{t} d t_{2} \exp \left\{-i\left(E_{k+q}-\omega_{q}\right) t_{2}\right\} G_{k k_{1}}\left(t_{2}\right)
$$




$$
P_{k k_{1}}^{(1)}=i \exp \left\{i\left(E_{k+q}+\omega_{q}\right) t\right\} \int_{0}^{t} d t_{2} \exp \left\{-i\left(E_{k+q}+\omega_{q}\right) t_{2}\right\} G_{k k_{1}}\left(t_{2}\right)
$$

Differentiating (38) and (39) with respect to time we have

$$
\begin{array}{ll}
i \frac{d}{d t} P_{k k_{1} q}=-\left(E_{k+q}-\omega_{q}\right) P_{k k_{1} q}-G_{k k_{1}}, & P_{k k_{1} q}(0)=0, \\
i \frac{d}{d t} P_{k k_{1} q}^{(1)}=-\left(E_{k+q}+\omega_{q}\right) P_{k k_{1} q}^{(1)}-G_{k k_{1}}, & P_{k k_{1} q}^{(1)}(0)=0 .
\end{array}
$$

Having solved the obtained system of three linear equations with the use of time Fourier transform we finally have

$$
G_{k k_{1}}(E)=\frac{1}{2 \pi} G_{k}^{0} \delta_{k k_{1}}\left[E-E_{k}-M_{k}(E)\right]^{-1},
$$

where

$$
M_{k}(E)=\sum A_{q}^{2}\left[\frac{N_{q}+1}{E-E_{k+q}-\omega_{q}}+\frac{N_{q}}{E-E_{k+q}+\omega_{q}}\right] .
$$

This result completely coincides with the result obtained in [2]. There use was made of a constant self-consistent field and traditional simultaneous decoupling.

The other limiting case is a strong dynamical coupling and the condition

$$
\left(A_{q n} / \omega_{q}\right)=\gamma_{q n}>>1 .
$$

In this case it is convenient to use Fröhlich Hamiltonian in discrete presentation:

$$
H=\sum E_{n} a_{n}^{+} a_{n}+I \sum a_{n+g}^{+} a_{n}+\sum \omega_{q} b_{q}^{+} b_{q}+\sum A_{q n} r_{q} a_{n}^{+} a_{n} .
$$

If the last term in expression (40) is large, the second term of the Hamiltonian can be neglected. As a result the Hamiltonian takes the form similar to Matsubara model for which our approximation gives exact results. In what follows use is made of the perturbation theory with respect to transfer integral. Notice that in the limit of very strong dynamical coupling any other regular approaches do not enable getting an exact solution.

\section{Investigation of Yukawa model}

The well-known Yukawa model describes interaction of two nucleons (Fermi particles) separated by a distance $r$ and interacting due to exchange by mesons (bosons). The case of different nucleons - a proton and a neutron (i.e. deutron) is considered in [23]. This model is similar to Matsubara one. However it includes not 
one but two particles occurring at different distances. Hamiltonian of this system takes the form:

$$
H=\sum \omega_{q} b_{q}^{+} b_{q}+\sum A_{q}^{(n)} r_{q} a_{n}^{+} a_{n}
$$

where

$$
\omega_{q}=\left(\mu^{2}+q^{2}\right)^{\frac{1}{2}}, A_{q}^{(n)}=g\left[2(2 \pi)^{3} \omega_{q}\right]^{-\frac{1}{2}} \exp \left\{-\frac{i}{2}(-1)^{n} q r\right\}
$$

$a_{n}^{+}, a_{n}$ are operators of the creation and annihilation of nucleons, $b_{q}^{+}, b_{q}$ are the operators of the creation and annihilation of mesons, $\mu$ is the meson mass, $g$ is the interaction parameter. A similar Hamiltonian also describes binding of two fermion or paulion states with one another due to their interaction with the boson field. Therefore it is straightforwardly related to the superconductivity problem.

One way to solve this problem is our method of approximate pair correlations which enables one to solve the problem exactly. It is convenient to base ourselves on paper [12] where an exact equation for the transition amplitude is obtained. The main aim of this section is to test once again the quality of our approximation on various models. Therefore, following paper [12], we will try to get equations for the transition amplitude (rather than for $\mathrm{CF}$, as it was done herein above). Let us consider the amplitude

$$
\varphi(1,2)=\left\langle 0\left|T a_{1}\left(t_{1}\right) a_{2}\left(t_{2}\right)\right| k\right\rangle
$$

at zero temperature. Here $|k\rangle=a_{1}^{+} a_{2}^{+}|0\rangle$, and suppose $t_{1}>t_{2}$. Let us write down motion equations for the amplitudes $\varphi(1,2)$ :

$$
\begin{aligned}
i \frac{\partial}{\partial t_{1}} \varphi(1,2) & =\sum_{q} A_{q}^{(1)}\left\langle 0\left|T a_{1}\left(t_{1}\right) r_{q}\left(t_{1}\right) a_{2}\left(t_{2}\right)\right| k\right\rangle \equiv \sum_{q} A_{q}^{(1)} M_{q}\left(t_{1} ; t_{1}, t_{2}\right) \varphi(1,2), \\
i \frac{\partial}{\partial t_{2}} \varphi(1,2)=\sum_{q} A_{q}^{(2)}\left\langle 0\left|T a_{1}\left(t_{1}\right) r_{q}\left(t_{2}\right) a_{2}\left(t_{2}\right)\right| k\right\rangle & \equiv \sum_{q} A_{q}^{(2)} M_{q}\left(t_{2} ; t_{1}, t_{2}\right) \varphi(1,2) .
\end{aligned}
$$

The last identical equality in this expression is a definition of $M_{q}$. For this quantity, holds

$$
M_{q}\left(t_{1} ; t_{1}, t_{2}\right)=\left\langle 0\left|r_{q}\left(t_{i}\right)\right| k\right\rangle+\frac{\delta}{\delta u_{q}\left(t_{i}\right)} \ln \varphi(1,2)
$$

We also have

$$
D_{q q_{1}}\left(t, t_{j} ; t_{1}, t_{2}\right)=\delta M_{q}\left(t_{j} ; t_{1}, t_{2}\right) / \delta u_{q_{1}}(t)
$$


To determine $M_{q}$ let us use differential equation

$$
\begin{gathered}
i \frac{\partial}{\partial t_{1}}\left\langle 0\left|T a_{1}\left(t_{1}\right) b_{q}^{ \pm}\left(t_{1}\right) a_{2}\left(t_{2}\right)\right| k\right\rangle=i \frac{\partial}{\partial t_{1}}\left[M_{q}^{ \pm}\left(t_{1} ; t_{1}, t_{2}\right) \phi(1,2)\right]= \\
=\mp \omega_{q} M_{q}^{ \pm}\left(t_{1} ; t_{1}, t_{2}\right) \phi(1,2) \mp\langle 0| T a_{1}\left(t_{1}\right)\left[A_{q}^{(1)} a_{1}^{+}\left(t_{1}\right) a_{1}\left(t_{1}\right)+\right. \\
\left.+A_{q}^{(2)} a_{2}^{+}\left(t_{1}\right) a_{2}\left(t_{1}\right)\right] a_{2}\left(t_{2}\right)|k\rangle+ \\
+\sum_{q_{1}} A_{q}^{(1)}\left\langle 0\left|T a_{1}\left(t_{1}\right) b_{q}^{ \pm}\left(t_{1}\right) r_{q_{1}}\left(t_{1}\right) a_{2}\left(t_{2}\right)\right| k\right\rangle .
\end{gathered}
$$

Since for the model under consideration

$$
a_{n}^{+}\left(t_{1}\right) a_{n}\left(t_{1}\right)=a_{n}^{+}\left(t_{2}\right) a_{n}\left(t_{2}\right)=a_{n}^{+}(0) a_{n}(0), \quad a_{1}\left(t_{1}\right) a_{1}\left(t_{1}\right)=a_{2}\left(t_{2}\right) a_{2}\left(t_{2}\right)=0,
$$

then, bearing in mind the equation for the amplitude $\varphi(1,2)$ and equality

$$
\begin{gathered}
\left\langle 0\left|T a_{1}\left(t_{1}\right) b_{q}^{ \pm}\left(t_{1}\right) r_{q_{1}}\left(t_{1}\right) a_{2}\left(t_{2}\right)\right| k\right\rangle= \\
=\left[M_{q}^{ \pm}\left(t_{1} ; t_{1}, t_{2}\right) M_{q_{1}}\left(t_{1} ; t_{1}, t_{2}\right)+D_{q}^{ \pm}\left(t_{1}-t_{2}\right) \delta\left(q+q_{1}\right)\right] \varphi(1,2),
\end{gathered}
$$

where

$$
M_{q}\left(t_{1} ; t_{1}, t_{2}\right)=M_{q}^{-}\left(t_{1} ; t_{1}, t_{2}\right)+M_{q}^{+}\left(t_{1} ; t_{1}, t_{2}\right) .
$$

With regard to (42), expression (43), finally yields

$$
\begin{gathered}
i \frac{\partial}{\partial t_{1}} M_{q}^{-}\left(t_{1} ; t_{1}, t_{2}\right)=\omega_{q} M_{q}^{-}\left(t_{1} ; t_{1}, t_{2}\right)+A_{-q}^{(1)}+A_{q}^{(1)}, \\
i \frac{\partial}{\partial t_{1}} M_{q}^{+}\left(t_{1} ; t_{1}, t_{2}\right)=-\omega_{q} M_{q}^{+}\left(t_{1} ; t_{1}, t_{2}\right)+A_{-q}^{(1)} .
\end{gathered}
$$

Solution of equations (44) has the form

$$
\begin{gathered}
M_{q}^{-}\left(t_{1} ; t_{1}, t_{2}\right)=M_{q}^{-}\left(0 ; 0, t_{2}\right) \exp \left\{-i \omega_{q} t_{1}\right\}-\left(A_{-q}^{(1)}+A_{q}^{(1)}\right) / \omega_{q} \\
M_{q}^{+}\left(t_{1} ; t_{1}, t_{2}\right)=M_{q}^{+}\left(0 ; 0, t_{2}\right) \exp \left\{i \omega_{q} t_{1}\right\}-A_{-q}^{(1)} / \omega_{q} .
\end{gathered}
$$

Functions

$$
D_{q}^{ \pm}\left(t_{1}-t_{2}\right)=\delta M_{q}^{ \pm}\left(t_{2} ; t_{1}, t_{2}\right) / \delta u_{q}\left(t_{1}\right)
$$

depend on the time difference. This fact results from the linear character of the interaction between fermions and bosons. To get a final solution we should determine how the integration constants $M_{q}^{ \pm}\left(0 ; 0, t_{2}\right)$ in expression (45) depend on the second oscillatory time $t_{2}$. Let us use the equations 


$$
\begin{gathered}
i \frac{\partial}{\partial t_{2}}\left\langle 0\left|T a_{2}(0) a_{2}\left(t_{2}\right)\right| k\right\rangle=\sum A_{q}^{(2)} a_{1}^{+}(0) r_{q}\left(t_{2}\right) a_{2}\left(t_{2}\right)|k\rangle \\
i \frac{\partial}{\partial t_{2}}\left\langle 0\left|T a_{1}(0) b_{q}^{ \pm}(0) a_{2}\left(t_{2}\right)\right| k\right\rangle=i \frac{\partial}{\partial t_{2}}\left[M_{q}^{ \pm}\left(0 ; 0, t_{2}\right) \varphi(1,2)\right]= \\
=\left\langle 0\left|T a_{1}(0) b_{q}^{ \pm}(0) \sum A_{q_{1}}^{(2)} r_{q_{1}}\left(t_{2}\right) a_{2}\left(t_{2}\right)\right| k\right\rangle
\end{gathered}
$$

These equations yield

$$
\begin{array}{cc}
i \frac{\partial}{\partial t_{2}} M_{q}^{-}\left(0 ; 0, t_{2}\right)=A_{-q}^{(2)} \exp \left\{i \omega_{q} t_{2}\right\}, & M_{q}^{-}(0 ; 0,0)=-\left(A_{-q}^{(2)} / \omega_{q}\right) \sigma \\
i \frac{\partial}{\partial t_{2}} M_{q}^{+}\left(0 ; 0, t_{2}\right)=0, \quad M_{q}^{+}(0 ; 0,0)=0 .
\end{array}
$$

Solving these equations with regard to the initial conditions we have:

$$
\begin{gathered}
M_{q}^{-}\left(0 ; 0, t_{2}\right)=-\left(A_{-q}^{(2)} / \omega_{q}\right) \cdot \exp \left\{i \omega_{q} t_{2}\right\} \\
M_{q}^{+}\left(0 ; 0, t_{2}\right)=0 .
\end{gathered}
$$

Substitution of (46) into (45) yields

$$
\begin{aligned}
& M_{q}\left(t_{1} ; t_{1}, t_{2}\right)=M_{q}^{-}\left(t_{1} ; t_{1}, t_{2}\right)+M_{-q}^{+}\left(t_{1} ; t_{1}, t_{2}\right)=, \\
& =-\frac{A_{-q}^{(2)}}{\omega_{q}} \exp \left\{-i \omega_{q}\left(t_{1}-t_{2}\right)\right\}-\frac{A_{q}^{(1)}+2 A_{-q}^{(1)}}{\omega_{q}} .
\end{aligned}
$$

The second equation for determining $M_{q}$ takes the form:

$$
\begin{gathered}
i \frac{\partial}{\partial t_{2}}\left\langle 0\left|T a_{1}\left(t_{1}\right) b_{q}^{ \pm}\left(t_{2}\right) a_{2}\left(t_{2}\right)\right| k\right\rangle=i \frac{\partial}{\partial t_{2}}\left[M_{q}^{ \pm}\left(t_{2} ; t_{1}, t_{2}\right) \varphi(1,2)\right]= \\
=\mp \omega_{q} M_{q}^{ \pm}\left(t_{2} ; t_{1}, t_{2}\right) \phi(1,2) \mp\langle 0| T a_{1}\left(t_{1}\right) a_{2}\left(t_{2}\right)\left[A_{q}^{(1)} a_{1}^{+}\left(t_{2}\right) a_{1}\left(t_{2}\right)+\right. \\
\left.+A_{q}^{(2)} a_{2}^{+}\left(t_{2}\right) a_{2}\left(t_{2}\right)\right]|k\rangle+ \\
+\left\langle 0\left|T a_{1}\left(t_{1}\right) b_{q}^{ \pm}\left(t_{2}\right) \sum_{q_{1}} A_{q_{1}}^{(2)} r_{q_{1}}\left(t_{2}\right) a_{2}\left(t_{2}\right)\right| k\right\rangle .
\end{gathered}
$$

With regard to (42) expression (48) yields 


$$
\begin{gathered}
i \frac{\partial}{\partial t_{2}} M_{q}^{-}\left(t_{2} ; t_{1}, t_{2}\right)=\omega_{q} M_{q}^{-}\left(t_{2} ; t_{1}, t_{2}\right)+A_{-q}^{(2)}+A_{q}^{(2)} \\
i \frac{\partial}{\partial t_{2}} M_{-q}^{+}\left(t_{2} ; t_{1}, t_{2}\right)=-\omega_{q} M_{-q}^{+}\left(t_{2} ; t_{1}, t_{2}\right)-A_{-q}^{(1)}-A_{-q}^{(2)} .
\end{gathered}
$$

Solutions of these equations have the form

$$
\begin{aligned}
& M_{q}^{-}\left(t_{2} ; t_{1}, t_{2}\right)=M_{q}^{-}\left(0 ; t_{1}, 0\right) \exp \left\{-i \omega_{q} t_{2}\right\}-\left(A_{-q}^{(2)}+A_{q}^{(2)}\right) \frac{1}{\omega_{q}} \\
& M_{-q}^{+}\left(t_{2} ; t_{1}, t_{2}\right)=M_{-q}^{+}\left(0 ; t_{1}, 0\right) \exp \left\{i \omega_{q} t_{2}\right\}-\left(A_{-q}^{(1)}+A_{-q}^{(2)}\right) \frac{1}{\omega_{q}} .
\end{aligned}
$$

To determine the integration constants $M_{q}^{ \pm}\left(0 ; t_{1}, 0\right)$ let us use the equations

$$
\begin{gathered}
i \frac{\partial}{\partial t_{1}}\left\langle 0\left|T a_{2}(0) a_{2}\left(t_{2}\right)\right| k\right\rangle=i \frac{\partial}{\partial t_{1}} \varphi(1,0)=\sum A_{q}^{(1)} a_{1}^{+}\left(t_{1}\right) r_{q}\left(t_{1}\right) a_{2}(0)|k\rangle \\
i \frac{\partial}{\partial t_{1}}\left\langle 0\left|T a_{1}\left(t_{1}\right) b_{q}^{ \pm}(0) a_{2}(0)\right| k\right\rangle=i \frac{\partial}{\partial t_{1}}\left[M_{q}^{ \pm}\left(0 ; t_{1}, 0\right) \varphi(1,0)\right]= \\
=\left\langle 0\left|T a_{1}\left(t_{1}\right) \sum_{q_{1}} A_{q_{1}}^{(1)} r_{q_{1}}\left(t_{1}\right) b_{q}^{ \pm}(0) a_{2}(0)\right| k\right\rangle .
\end{gathered}
$$

from which it follows that

$$
\begin{gathered}
i \frac{\partial}{\partial t_{1}} M_{q}^{-}\left(0 ; t_{1}, 0\right)=0, \quad M_{q}^{-}(0 ; 0,0)=0 \\
i \frac{\partial}{\partial t_{1}} M_{-q}^{+}\left(0 ; t_{1}, 0\right)=A_{-q}^{(1)} e^{-i \omega_{q} t_{1}}, \quad M_{-q}^{+}(0 ; 0,0)=A_{-q}^{(1)} e^{-i \omega_{q} t_{1}} \frac{1}{\omega_{q}} .
\end{gathered}
$$

These equations with regard to the initial conditions have solutions of the form

$$
M_{q}^{-}\left(0 ; t_{1}, 0\right)=0, \quad M_{-q}^{+}\left(0 ; t_{1}, 0\right)=A_{-q}^{(1)} e^{-i \omega_{q} t_{1}} \frac{1}{\omega_{q}} .
$$

Therefore we have:

$$
\begin{aligned}
& M_{q}\left(t_{2} ; t_{1}, t_{2}\right)=M_{q}^{-}\left(t_{2} ; t_{1}, t_{2}\right)+M_{-q}^{+}\left(t_{2} ; t_{1}, t_{2}\right)= \\
= & \frac{A_{-q}^{(2)}}{\omega_{q}} \exp \left\{-i \omega_{q}\left(t_{1}-t_{2}\right)\right\}-\frac{2 A_{-q}^{(2)}+A_{q}^{(1)}+A_{-q}^{(1)}}{\omega_{q}} .
\end{aligned}
$$

Now we can return back to system (42). Having substituted therein the results obtained we get: 


$$
\begin{gathered}
i \frac{\partial}{\partial t_{1}} \varphi(1,2)=\sum_{q} A_{q}^{(1)}\left(-\frac{A_{-q}^{(2)}}{\omega_{q}} e^{-i \omega_{q} t}-\frac{A_{q}^{(1)}+2 A_{-q}^{(1)}}{\omega_{q}}\right) \varphi(1,2) \\
i \frac{\partial}{\partial t_{2}} \varphi(1,2)=\sum_{q} A_{q}^{(2)}\left(-\frac{A_{-q}^{(1)}}{\omega_{q}} e^{-i \omega_{q} t}-\frac{A_{q}^{(2)}+2 A_{-q}^{(2)}+A_{-q}^{(1)}}{\omega_{q}}\right) \varphi(1,2) .
\end{gathered}
$$

where $t=t_{1}-t_{2}$.

Noting that by condition of symmetry

$$
\sum A_{q}^{(1)}\left[A_{q}^{(1)}+2 A_{-q}^{(1)}\right]=\sum A_{q}^{(2)}\left[A_{q}^{(2)}+2 A_{-q}^{(2)}\right]=-\varepsilon
$$

we choose the starting point of the energy so that $\varepsilon=0$. Then with regard to the explicit form of $A_{q}^{(n)}$ presented in (41), we can rewrite (50) as

$$
\begin{gathered}
i \frac{\partial}{\partial t_{1}} \varphi(1,2)=-\frac{\lambda}{4 \pi} \sum_{q} \frac{e^{i q r}}{\omega_{q}^{2}} e^{-i \omega_{q} t} \varphi(1,2) \\
i \frac{\partial}{\partial t_{2}} \varphi(1,2)=\left[\frac{\lambda}{4 \pi} \sum_{q} \frac{e^{-i q r}}{\omega_{q}^{2}} e^{-i \omega_{q} t}-V(r)\right] \varphi(1,2),
\end{gathered}
$$

where

$$
\lambda=\frac{g^{2}}{(2 \pi)^{2}}, \quad V(r)=\sum \frac{e^{-i q r}}{2(2 \pi)^{3} \omega_{q}^{2}}=\int d^{3} q \frac{e^{-i q r}}{2(2 \pi)^{3}\left(\mu^{2}+q^{2}\right)}=\frac{1}{4 \pi} \frac{e^{-\mu r}}{r} .
$$

Having expressed the phase in the explicit form we can present the quantity $\varphi(1,2)$ as

$$
\varphi(1,2)=\varphi(t) \exp \left(-i E \frac{t_{1}+t_{2}}{2}\right) .
$$

Besides, let us introduce the new variables $x=(i t / r)$ and a function

$$
g(x)=\frac{1}{4 \pi} \sum \frac{e^{i q r}}{\omega_{q}^{3}} e^{-\omega_{q} r x}
$$

Now we can rewrite (51) in the form of Bethe-Salpeter equation

$$
\begin{gathered}
-\frac{\partial}{\partial t_{1}} \frac{\partial}{\partial t_{2}} \varphi(1,2)= \\
=\left(\frac{\lambda}{4 \pi} \sum \frac{e^{i q r}}{\omega_{q}} e^{-i \omega_{q} t}+\lambda^{2} V(r) \frac{1}{4 \pi} \sum \frac{e^{i q r}}{\omega_{q}^{2}} e^{-i \omega_{q} t}-\lambda^{2} \frac{1}{(4 \pi)^{2}}\left[\sum \frac{e^{i q r}}{\omega_{q}^{2}} e^{-i \omega_{q} t}\right]^{2}\right) \varphi(1,2)
\end{gathered}
$$


or, in terms of variables $x$, as a one-dimensional Schröedinger equation:

$$
\left(-\frac{d^{2}}{d x^{2}}+U(x)\right) \phi(x)=-\frac{(E r)}{4} \phi(x)
$$

with potential

$$
U(x)=-\lambda^{2} \frac{d^{2} g(x)}{d x^{2}}+\lambda^{2} \pi e^{-\mu r} \frac{d g(x)}{d x}+\lambda^{2}\left(\frac{d g(x)}{d x}\right)^{2} .
$$

Equation (52) was also obtained in [12] by summation of all the orders of the perturbation theory. This coincidence makes it clear that our method provides exact results for Yukawa model. The "wave" function $\varphi(x)$ should satisfy natural boundary conditions $\varphi(\infty)=0$ and $\varphi^{\prime}(0)=0$. Therefore $\varphi(x)$ differs from zero only for some very special values of $E$. As a result the energy $E$ (or the mean distance between nucleons) assumes quantum values.

For phonons $\mu=0$ and expression (53) takes on the form

$$
U(x)=-\frac{1}{1+x^{2}}-\lambda^{2} \cdot \pi \cdot \operatorname{arcctg}(x)+\lambda^{2} \cdot(\operatorname{arcctg}(x))^{2}
$$

This problem was solved exactly in paper [12]. Nevertheless, some researchers used such an approximation as partial summation of Feynman diagrams [13]. It should be noted that in the case of summation of ladder approximation we can obtain the expression [13]

$$
U(x)=-\frac{1}{1+x^{2}} .
$$

The solution of equation (52) considerably depends on the behavior of the potential as $x \rightarrow \infty$. This behavior is different in the cases (54) and (55). The difference in asymptotics leads to different eigenvalues of quantities.

The results obtained suggest that the methods of a constant field and restricted diagram summation (for example in ladder approximation) are invalid for solving the problems of the type being considered. In this case it is appropriate to use our approximation of pair correlations which enables getting an exact result.

\section{Method of pair correlations for systems with frequency effect}

Systems with frequency effect are more sophisticated than those with linear interaction. Let us consider a two-level model with the Hamiltonian:

$$
H=\sum H_{n} a_{n}^{+} a_{n}+\sum I_{m n} a_{m}^{+} a_{n} \quad m, n=1,2
$$


where

$$
H_{n}=E_{n}+\sum A_{q n} r_{q}+\sum B_{q n} r_{q}^{2}+\sum \omega_{q} b_{q}^{+} b_{q} .
$$

Let us calculate $\mathrm{CF}$ of the form

$$
G_{m n}(t)=<a_{n}^{+}(0) a_{m}(0) a_{m}^{+}(t) a_{n}(t)>,
$$

in terms of which the transition probability is expressed. The motion equation for this $\mathrm{CF}$ is

$$
\begin{aligned}
i \frac{d}{d t} G_{m n}(t) & =\left[E_{n}-E_{m}+\sum\left(\left(A_{q n}-A_{q m}\right) \Delta_{q}(t)+\left(B_{q n}-B_{q m}\right) \Delta_{q}^{2}(t)\right)\right] G_{m n}(t)- \\
& -\sum I_{p m} G_{p n}+\sum I_{p n} G_{m p} \quad G_{m n}(0)=<a_{m}^{+} a_{m}>\delta_{m n},
\end{aligned}
$$

where

$$
\Delta_{q}(t)=<r_{q}(t)>+\delta / \delta u_{q} .
$$

Solution of equation (56) will be searched for in the form of the product:

$$
G_{m n}(t)=G_{m n}(0) g_{m n}(t, u) \exp \left\{S_{m n}(t, u)\right\}
$$

where $\exp \left\{S_{m n}(t, u)\right\}$ is a solution of equation (56) for $I_{m n}=0$. Having substituted (57) into (56) and performed integration we get for functional $g_{m n}(t, u)$ a system of linear equations:

$$
g_{m n}(t, u)=g_{m n}(0, u)-i \int_{0}^{t} e^{-S_{m n}(t)}\left[\sum I_{p m} e^{S_{p m}\left(t_{1}\right)} g_{p n}\left(t_{1}\right)-\sum I_{n p} e^{S_{m p}\left(t_{1}\right)} g_{m p}\left(t_{1}\right)\right] d t_{1}
$$

that can serve as the basis for developing the perturbation theory so that to calculate $\mathrm{G}_{m n}(t)$. After that it remains to determine $S_{m n}(t, u)$. From (56) we have:

$$
\begin{gathered}
i \frac{d}{d t} S_{m n}(t)=E_{n}-E_{m}+\sum\left(\left(A_{q n}-A_{q m}\right) M_{q}(t ; t, m n)+\right. \\
\left.+\left(B_{q n}-B_{q m}\right)\left[M_{q}^{2}(t ; t, m n)+D_{q q}(t, t ; t, m n)\right]\right)
\end{gathered}
$$

where we introduced new functions $M_{q}(t ; t, m n), D_{q q}(t, t ; t, m n)$ such that

$$
\begin{aligned}
& M_{q}\left(t_{1} ; t, m n\right)=<r_{q}\left(t_{1}\right)>+\delta S_{m n}(t) / \delta u_{q}\left(t_{1}\right) \\
& D_{q q_{1}}\left(t_{2}, t_{1} ; t, m n\right)=\delta M_{q}\left(t_{1} ; t, m n\right) / \delta u_{q_{1}}\left(t_{2}\right) .
\end{aligned}
$$

Now solution of (59) will be written as 


$$
\begin{gathered}
S_{m n}(t)=-i\left[\left(E_{n}-E_{m}\right) t+\sum\left(\left(A_{q n}-A_{q m}\right) \int_{0}^{t} d t_{1} M_{q}\left(t_{1} ; t_{1}, m n\right)+\right.\right. \\
\left.\left.+\left(B_{q n}-B_{q m}\right) \int_{0}^{t} d t_{1}\left[M_{q}^{2}\left(t_{1} ; t_{1}, m n\right)+D_{q q}\left(t_{1}, t_{1} ; t_{1}, m n\right)\right]\right)\right] .
\end{gathered}
$$

Taking (60) into account, let us rewrite the expression for $M_{q}(t ; t, m n)$ in a new form:

$$
\begin{gathered}
M_{q}\left(t_{1} ; t, m n\right)=<r_{q}\left(t_{1}\right)>-i \int_{0}^{t} d t_{2}\left[\left(A_{q n}-A_{q m}\right) D_{q q}\left(t_{1}, t_{2} ; t_{2}, m n\right)+\right. \\
\left.+2\left(B_{q n}-B_{q m}\right) M_{q}\left(t_{2} ; t_{2}, m n\right) D_{q q}\left(t_{1}, t_{2} ; t_{2}, m n\right)\right]
\end{gathered}
$$

Expression (60) can also be presented as

$$
\begin{gathered}
S_{m n}(t)=-i\left[\left(E_{n}-E_{m}\right) t+\frac{1}{2} \sum\left(\left(A_{q n}-A_{q m}\right) \int_{0}^{t} d t_{1} M_{q}\left(t_{1} ; t, m n\right)+\right.\right. \\
\left.\left.+\left(B_{q n}-B_{q m}\right) \int_{0}^{t} d t_{1} D_{q q}\left(t_{1}, t_{1} ; t_{1}, m n\right)\right)\right] .
\end{gathered}
$$

In order to make sure that it is true it suffices to use the equality

$$
\begin{gathered}
\frac{1}{2}\left(A_{q n}-A_{q m}\right) \int_{0}^{t} M_{q}\left(t_{2} ; t, m n\right) d t_{1}=\left(A_{q n}-A_{q m}\right) \int_{0}^{t} M_{q}\left(t_{1} ; t_{1}, m n\right) d t_{1}+ \\
+\left(B_{q n}-B_{q m}\right) \int_{0}^{t} M_{q}^{2}\left(t_{1} ; t_{1}, m n\right) d t_{1},
\end{gathered}
$$

which is easily proved by integration of expression (61). Bearing in mind that

$$
M_{q}\left(t_{2} ; t, m n\right)=-i\left(A_{q n}-A_{q m}\right) \int_{0}^{t} D_{q q}\left(t_{1}, t_{2} ; t, m n\right) d t_{2},
$$

we have:

$$
\begin{gathered}
S_{m n}(t)=-i\left(E_{n}-E_{m}\right) t- \\
\frac{1}{2} \sum\left(\left(A_{q n}-A_{q m}\right)\left(A_{q_{1} n}-A_{q_{1} m}\right) \int_{0}^{t} \int_{0}^{t} d t_{1} d t_{2} D_{q q_{1}}\left(t_{1}, t_{2} ; t, m n\right)-\right. \\
\left.-i \sum\left(B_{q n}-B_{q m}\right) \int_{0}^{t} d t_{1} D_{q q}\left(t_{1}, t_{1} ; t_{1}, m n\right)\right)
\end{gathered}
$$


Exact presentation of (62) is a generalization of a well-known expression for the constant of the electron transfer rate in the case of a nonlinear interaction. Potentialities of this presentation become especially apparent in considering systems with continuous spectrum. In this case, as distinct from other approaches, the influence of a nonlinear interaction on the transition probability can be investigated most carefully.

This expression is also valid in the case when $B$ is a nondiagonal matrix, i.e. upon rotation of normal oscillation coordinates in the course of the reaction (Dushinsky effect). More thoroughly this point is considered in [24]. To get an equation for $D$ let us take a variational derivative of $M_{q}\left(t_{1} ; t, m n\right)$ in expression (61). We will have

$$
D_{q q}\left(t_{1}, t_{2} ; t, m n\right)=D_{q q}^{0}\left(t_{1}-t_{2}\right)-2 i\left(B_{q n}-B_{q m}\right) \int_{0}^{t} d t_{3} D_{q q}\left(t_{2}, t_{3} ; t_{3}, m n\right) D_{q q}\left(t_{1}, t_{3} ; t_{3}, m n\right)
$$

To make the formulae less cumbersome without losing the essence of the problem let us consider a particular case when Hamiltonian has the form

$$
H=H_{1} a_{1}^{+} a_{1}+H_{2} a_{2}^{+} a_{2}+I_{12}\left(a_{1}^{+} a_{2}+a_{2}^{+} a_{1}\right)
$$

where

$$
H_{1}=E_{1}+B_{1} r^{2}+\sum \omega_{q} b_{q}^{+} b_{q} ; \quad H_{2}=E_{2}+B_{2} r^{2}+\sum \omega_{q} b_{q}^{+} b_{q} .
$$

In this case

$$
S_{12}(t)=-i\left(E_{1}-E_{2}\right) t-i\left(B_{1}-B_{2}\right) \int_{0}^{t} d t_{1} D\left(t_{1}, t_{1} ; t_{1}, 12\right) .
$$

where

$$
D_{q q_{1}}\left(t_{2}-t_{1}\right)=\frac{\delta<r_{q}\left(t_{1}\right)>}{\delta u_{q_{1}}\left(t_{2}\right)} \text {. }
$$

and

$$
\begin{gathered}
G(t)=G(0) \exp \left\{-i\left(E_{1}-E_{2}\right) t-i\left(B_{1}-B_{2}\right) \int_{0}^{t} d t_{1} D\left(t_{1}, t_{1} ; t_{1}, 12\right)\right\} \\
D\left(t_{2}, t_{1} ; t, 12\right)=D^{0}\left(t_{2}-t_{1}\right)-2 i\left(B_{1}-B_{2}\right) \int_{0}^{t} d t_{3} D\left(t_{2}, t_{3} ; t_{3}, 12\right) D\left(t_{1}, t_{3} ; t_{3}, 12\right) .
\end{gathered}
$$

Function $D^{0}(t)$ for the oscillator subsystem is equal to 


$$
D^{0}(t)=\left[N e^{i \omega t}+(N+1) e^{-i \omega t}\right], \quad t \geq 0 .
$$

This quantity was used in Section 3 devoted to Fröhlich model [25-28]. In this section concerned with transitions in media with nonlinear interaction we will consider transitions in high-viscosity liquids where oscillations are impossible [25, 26]. The expression for $D^{0}$ in such media will be written as

$$
D^{0}\left(t_{2}-t_{1}\right)=d^{2} \exp \left\{-\omega\left|t_{2}-t_{1}\right|\right\}, \quad t_{2}>t_{1} .
$$

To solve integral equation (64), we present $D\left(t_{2}, t_{1} ; t, 12\right)$ as

$$
D\left(t_{2}, t_{1} ; t, 12\right)=d^{2} \exp \left\{-\omega t_{2}+\omega t_{1}\right\} \varphi(t) \quad t_{2}>t_{1} .
$$

Then from integral equation (64) we have

$$
\varphi(t)=1-2 i\left(B_{1}-B_{2}\right) d^{2} \int_{0}^{t} \exp \left\{-2 \omega t+2 \omega t_{3}\right\} \varphi^{2}\left(t_{3}\right) d t_{3} .
$$

Differentiating this expression with respect to time we get

$$
\frac{d \varphi(t)}{d t}=2 \omega-2 \omega \varphi(t)-2 i\left(B_{1}-B_{2}\right) d^{2} \varphi^{2}(t) .
$$

Solution of this equation has the form

$$
\varphi(t)=\frac{2}{2 i\left(B_{1}-B_{2}\right) d^{2}}\left[-\omega+\frac{d}{d t}\left[\ln \left(e^{\Omega t}+C e^{-\Omega t}\right)\right],\right.
$$

where

$$
\Omega=\left(\omega^{2}+4 i\left(B_{1}-B_{2}\right) d^{2} \omega\right)^{1 / 2}, \quad C=\frac{\Omega-\omega+2 i\left(B_{1}-B_{2}\right) d^{2}}{\Omega+\omega-2 i\left(B_{1}-B_{2}\right) d^{2}} .
$$

Having substituted $\varphi(t)$ into (63) and performed integration we obtain the exact result as it was done in [24]

$$
G(t)=G(0) \exp \left\{i\left(E_{1}-E_{2}\right) t\right\} \cdot\left[\frac{|1+C|}{\left|e^{\Omega t}+C e^{-\Omega t}\right| e^{-\omega t}}\right]^{1 / 2} .
$$

It would take enormous effort to get this result by traditional summation of Feynman diagrams. It is yet more proof that our method is more advantageous as compared to the method of diagrams and other widely used approximations. 


\section{Conclusion}

We have described in detail a universal method for considering a dynamical coupling and other interactions in quantum-mechanical systems. This method has a wide range of physical and physicochemical applications.

The paper is based on the idea that it is in principle possible to reduce an infinite system of linear equations for correlation functions to a finite nonlinear one. This makes possible truncation of Bogolubov's chains. The physical meaning of the approximation suggested is that an alternating self-consistent field is introduced. The method enables one to get exact solutions to well-known models of molecular systems.

The possibility of effective truncation of Bogolubov's chains is demonstrated by the example of Matsubara, Yukawa and Froehlich models with zero band for nonlinear and quadratic electron-phonon interaction. In all the cases the method yields true results coinciding with those obtained earlier with the use of other approaches. A necessary condition for the exact solution is a small probability of triple and higher-order correlations.

Though higher CFs were not taken into account in the above-presented method for closing chains, it is just this approach that enables one to consider them. No other methods can do this. Our method enables one to take account of any CF determined preliminarily. Their consideration can be especially effective in the studies of nuclear matter - in the case of light nuclei.

A similar approach was suggested by Peletminsky school [8, 29-33]. The method is based on the introduction of an arbitrary effective coordinate and also makes possible reduced description of the system. In our method analogous coordinates are a result of calculations.

Our approach suits well to investigate energy and/or charge transfer in crystals and films. However it is especially promising for quasi-one-dimensional models of polymer chains. Just in such systems dynamical interactions prevail while triple correlations are excluded in fact even if neighboring loops of a polymer chain may come in contact. This application of our method has become especially actual in recent decades when a huge number of investigations of charge transfer in DNA have been carried out [34-37].

Critical reduction of the number of equations enables one to decrease the machine time consumption by several orders of magnitude. Hence the method suggested offers exciting possibilities for investigation of quantum models. Automatical exclusion of irrelevant correlations is rather a useful feature of our method. Just this property makes possible "automatisation" of the methods in the simplest console applications.

\section{Acknowledgments}

The work was done with the support from the Russian Foundation for Basic Research [Project № 16-07-00305-a]. 


\section{References}

1. Bogolyubov N.N., Tyablikov S.V. The belated and forestall Green function in statistical physics. // Doklady AN SSSR, 1959. V. 126, No. 1. PP. 53-56 (in Russian).

2. Zubarev D.N. Double-time Green functions in statistical physics. // Sov. Phys. Usp., 1960. V.3. PP. 320-345. - URL: https://ufn.ru/en/articles/1960/3/b/

3. Bonch-Bruevich V.L., Tyablikov S.V. The Green function method in statistical mechanics. Amsterdam: North-Holland, 1962. 251 p. URL: https://books.google.ru/books?isbn=0486797155

4. Zubarev D.N. Nonequilibrium statistical thermodynamics. Consultants Bureau, 1974. 439 p. URL: https://books.google.ru/books?isbn=030610895X

5. Bogolyubov N.N., Bogolyubov N.N. Jr. Introduction to quantum statistical mechanics. Worid Sci, 1982, 384 p. -

URL: https://books.google.ru/books?isbn=9814295825

6. Tyablikov S.V. Methods in the Quantum Theory of Magnetism. New York: Plenum Press, 1967. - URL: https://books.google.ru/books?isbn=0306302632

7. Zubarev D.N., Morozov V.G., Ropke G. Statistical Mechanics of Nonequilibrium Processes. Volume 1: Basic Concepts. Kinetic theory. Berlin, Akademie Verlag, 1996. - URL: https://books.google.ru/books?isbn=3055017080

8. Kovalevsky M.Yu., Peletminsky S.V. Statistical mechanics of quantum fluids and crystals, M.: Fizmatlit. 2006. 368 p. (in Russian).

9. Kubarev S.I., Ponomarev O.A. The effects of dynamic binding in statistical physics. Moscow: Nauka. 1992. 141 p.

10. Ponomarev O.A., Shigaev A.S., Zhukov A.I., Lakhno V.D. Hole Conductivity in Heterogeneous DNA Fragments. // Mathematical Biology \& Bioinformatics, 2013. V. 8. No 2. PP. 135-160. - URL: https://doi.org/10.17537/2013.8.135

11. Matsubara T. Note on the Generating Function of Two-Time Green Functions. // Prog. Theor. Phys., 1964. V. 32. No. 1. PP. 52-64. URL: https://doi.org/10.1143/PTP.32.50

12. Mugibayashi N. Exact treatment of the bound state problems in the nonrelativistic quantum field theory. // Progr. Theor. Phys., 1961. V. 25, No. 5. PP. 803821. - URL: https://doi.org/10.1143/PTP.25.803

13. Feynman R. P. Statistical mechanics, Chap. 2, California, Institute of technology, Massachusetts. 1972. -

URL: https://books.google.ru/books?isbn=0201360764

14. Ivanov A.B., Lomakin G.S., Ponomarev O.A. Investigation of systems with quadratic interaction by the method of functional derivatives. // Theor. and Math. Phys., 1979. V. 41, Part 2. PP. 1020-1028. -

URL: https://link.springer.com/article/10.1007/BF01028508

15. Kubarev S.I., Ponomarev O.A. The non-simultaneous decupling method for correlation functions. // Phys. Lett., 1968. V. 26A, No. 12. PP. 633-634. -

URL: https://doi.org/10.1016/0375-9601(68)90165-5 
16. Abrikosov A.A., Gorkov L.P., Dzyaloshinskii I.E. Methods of quantum field theory in statistical physics. Englewood Cliffs: Prentice-Hall. 1965. 365 p. -

URL: https://www.twirpx.com/file/2267252/

17. Polarons, ed. by Yu. A. Firsov, M:, Nauka, 1975, (in Russian). URL: https://www.twirpx.com/file/403788/

18. Ivanov A.I., Ponomarev O.A. Investigation of the Frohlich model by the method of functional derivatives. // Theor. And Math. Phys., 1977. V. 30, Part 3. PP. 246-255. - URL: https://link.springer.com/article/10.1007/BF01036718

19. Min S.K., Agostini F., Gross E.K.U. Coupled-Trajectory Quantum-Classical Approach to Electronic Decoherence in Non-adiabatic Processes. // Phys. Rev. Lett., 2015. V. 115, P. 073001.-

URL: https://journals.aps.org/prl/abstract/10.1103/PhysRevLett.115.073001

20. Wang L., Beljonne D. Flexible Surface Hopping Approach to Model the Crossover from Hopping to Band-like Transport in Organic Crystals. // J. Phys. Chem. Lett., 2013. V. 4. PP, 1888-1894. -

URL: https://doi.org/10.1021/jz400871j

21. Si W., Wu C.Q. Decoherence and energy relaxation in the quantum-classical dynamics for charge transport in organic semiconducting crystals: An instantaneous decoherence correction approach. // J. Chem. Phys., 2015. V. 143. P. 024103. URL: https://doi.org/10.1063/1.4926534

22. Lee J., Chung J. W., Kim D.H., Lee B. L., Park J. I., Lee S., Hausermann R., Batlogg B., Lee S. S., Choi I., Kim I. W., Kang M. S. Thin Films of Highly Planar Semiconductor Polymers Exhibiting Band-like Transport at Room Temperature, // (2015), J. Am. Chem. Soc., 2015. V. 137. P. 7990-7993. -

URL: https://doi.org/10.1021/jacs.5b04253

23. Lakhno V.D. Quantum model of deuteron. // Theoret. and Math. Phys., 1994. V. 100, Part 2. PP. 963-969. - URL: https://doi.org/10.1007/BF01016759

24. Bikbaev N. Rh., Ivanov A.I., Lomakin G.S., Ponomarev O.A. Theory of nonradiative processes in the "non-Condon" approximation. Sov. Phys. JETP., 1978. V. 47, No. 6. P. 1121-1127. - URL:

http://www.jetp.ac.ru/cgi-bin/dn/e_047_06_1121.pdf

25. Ponomarev O.A., Ponomareva V.A. Breit-Wigner resonance in chemistry. // Theoretical and Experimental Chemistry, 1990. V. 26, No. 4, PP. 398-406. - URL: https://doi.org/10.1007/BF00530252

26. Ponomarev O.A., Nazarov N.U. Influence of medium viscosity on adiabatic reaction rates. // Russian Journal of Electrochemistry, 1990, V. 26, No. 8. PP. 900909. - URL: https://elibrary.ru/item.asp?id=21456201

27. Nazarov N.U., Ponomarev O.A. Quantum dynamics of transitions in two-level electron-phonon systems with strong interactions. // Theor. and Math. Phys., 1990. V. 85, No. 1, P. 1072-1081. - URL: https://doi.org/10.1007/BF01017249

28. Nazarov N.U., Ponomarev O.A. The one-particle Green function for strongly correlated finite Fermi system. // Phys. Lett., 1992. V. 164. No. 5-6. PP. 447-450. URL: https://doi.org/10.1016/0375-9601(92)90112-Y 
29. Kovalevsky M.Yu., Rozhkov A.A. On the theory of disordered magnetics. // Physica A, 1995. V. 216. Issues 1-2. PP. 169-184. - URL:

https://doi.org/10.1016/0378-4371(94)00293-3

30. Kovalevsky M.Yu., Peletminsky S.V. Hydrodynamic theory of magnets with complete spontaneous symmetry breaking. // Theor. and Math. Phys., 1994. V. 100. PP. 846-856. - URL: https://doi.org/10.1007/BF01017322

31. Akhieser A.I., Isaev A.A., Peletminsky S.V., Rekalo A.P., Yatsenko A.A. Theory of superfluidity of nuclear matter based on the Fermi-liquid approach. // Zh. Eksp. Teor. Fiz., 1997. V. 112, PP. 3-24. - URL: https://doi.org/10.1134/1.558307

32. Peletminsky A.S., Peletminsky S.V., Slyusarenko Yu. V. Theory of a spatially periodic bose condensate in the weakly nonideal Bose gas model. // Theor. and Math. Phys., 2000. V. 125, Issue 1. PP. 1431-1453.-

URL: https://doi.org/10.1007/BF02551046

33. Peletminsky A.S., Peletminsky S.V., Slyusarenko Yu. V. Variational principle in the spatially periodic Bose condensate theory // Laser Physics, 2002. V. 12. P. 162187.

34. Conron S.M.M., Thazhathveetil A.K., Wasielewski M.R., Burin A.L., Lewis F.D. Direct measurement of the dynamics of hole hopping in extended DNA G-tracts. An unbiased random walk. // J. Am. Chem. Soc., 2010. V. 132, PP. 14388-14390. URL: https://doi.org/10.1021/ja106991f

35. Harris M.A., Mishra A.K., Young R.M., Brown K.E., Wasielewski M.R., Lewis F.D.. (2016). Direct observation of the hole carriers in DNA photoinduced charge transport. // J. Am. Chem. Soc. V. 138, PP. 5491-5494. -

URL: https://doi.org/10.1021/jacs.6b00702

36. Lewis F.D., Liu X., Liu J., Miller S.E., Hayes R.T., Wasielewski M.R. Direct measurement of hole transport dynamics in DNA // Nature, 2000. V. 406, PP. 51-53. - URL: https://doi.org/10.1038/35017524

37. Giese B., Biland A. Recent development of charge injection and charge transfer in DNA. // Chem. Commun., 2002. PP. 667-672. -

URL: http://dx.doi.org/10.1039/B111044F 


\section{Оглавление}

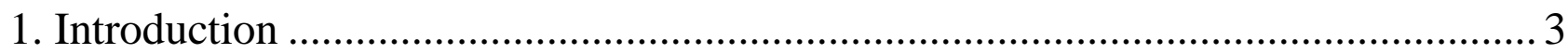

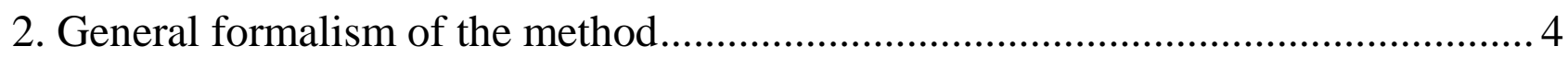

3. The use of the method for generalized Matsubara model ........................................ 10

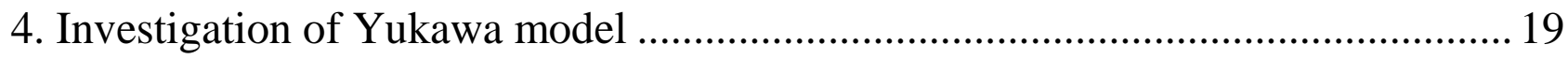

5. Method of pair correlations for systems with frequency effect .............................25

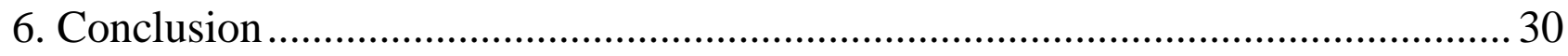

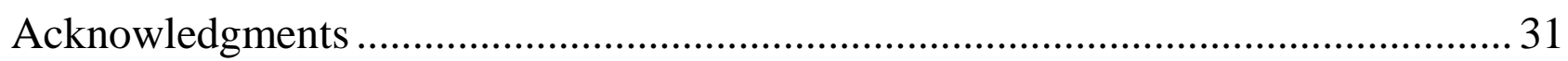

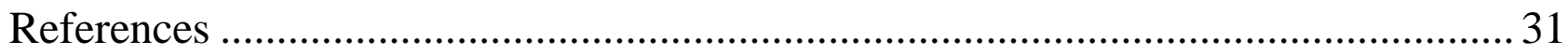

